\title{
Smart Structures: Model Development and Control Applications
}

\author{
Ralph C. Smith \\ Center for Research in Scientific Computation \\ Department of Mathematics \\ North Carolina State University \\ Raleigh, NC 27695-8205 \\ rsmith@eos.ncsu.edu
}

\begin{abstract}
This paper addresses aspects of model development and control design for smart structure which utilize piezoelectric, electrostrictive, magnetostrictive or shape memory alloys. The advent of these materials as sensors and actuators provides the opportunity for designing systems with improved performance while reducing weight, hardware and power requirements. At high drive levels, however, all of these materials exhibit nonlinear constitutive relations and varying degrees of hysteresis which must be accommodated to realize their full potential in smart structures. We outline a variety of modeling techniques and illustrate their use in feedback, feedforward and adaptive control designs.
\end{abstract}




\section{Introduction}

Increased demands for high performance control design in combination with recent advances in material science have produced a class of systems termed smart, intelligent or adaptive structures. While subtle differences may be associated with the individual terms, these structures are generally defined as systems whose dynamics can be monitored or modified by distributed sensors and actuators, in accordance with an integrated control law, to accommodate time-varying exogenous inputs or changing environmental conditions. Specific choices for the actuators, sensors and control laws are dictated by the design requirements for the system.

For aeronautic and aerospace systems, control transducers must be light- weight and should typically have minimal effect on the passive system dynamics. Furthermore, actuators must provide the required strain or force inputs using the available power supplies which, in certain aerospace structures, may require the scavenging of power from other components in the system. Restrictions on size and weight also dictate that transducers in some regimes must be capable of multiple roles. For example, the transducers which monitor and control vibrations in an aircraft fuselage may also be required to act as inputs and sensors for health monitoring or nondestructive evaluation of the structure. The limitations on the mass and size of transducers are often relaxed in industrial applications but output requirements may be more stringent. For example, the actuators employed for controlling vibrations in the cutting head of a milling machine are required to generate massive forces throughout a wide frequency range.

Actuators and sensors comprised of smart or active materials can meet these criteria. Like the definition for smart structures, the definitions of smart or active materials can vary somewhat between fields. We define active actuator materials as those which can convert electrical, magnetic or thermal energy to mechanical energy while sensor effects are provided by the opposite conversion of energy. In this discussion, we focus on piezoelectric, electrostrictive, magnetostrictive materials and shape memory alloys. The first three compounds can be used for both sensing and actuating while shape memory alloys are used only in the latter capacity.

Piezoelectric compounds have been the most widely used transducer for smart structure design due to the fact that they are lightweight and compact, relatively inexpensive, and exhibit fairly linear fieldstrain relations at low drive levels. Due to the inherent direct and converse piezoelectric effects, they can also be employed as both sensors and actuators. At higher drive levels, however, they exhibit hysteresis which must be accommodated in applications that require micropositioning or precise control design.

In certain applications, electrostrictive elements constructed from relaxor ferroelectric materials are advantageous over piezoceramic patches due to the fact that they exhibit minimal hysteresis when employed in the diffuse transition region near the material's bulk Curie temperature. This makes them advantageous in systems including sonar transducers and deformable mirrors. Unlike piezoelectric materials, electrostrictive compounds are not poled and hence exhibit few aging effects. As detailed in Section 2, however, the constitutive behavior of the materials is highly temperature-dependent and nonlinear near saturation. Both aspects must be accommodated when designing control systems which utilize the compounds.

The magnetic analogue of the electrostrictive compounds are magnetostrictive materials which convert magnetic energy to mechanical energy and vice-versa. Due to the circuits required to generate the driving magnetic fields, transducers which utilize magnetostrictive cores are currently larger and more massive than piezoelectric or electrostrictive patches. The giant forces and strains generated by the transducers, however, make them advantageous in certain industrial systems, and as material properties and transducer designs are refined, the scope of their application should rapidly increase. From a modeling and control perspective, the nonlinearities and hysteresis inherent to the materials at moderate to high drive levels must be accommodated before the materials can be utilized to their full potential. 
Shape memory alloys produce strains on the order of $10 \%$ in response to thermal inputs. This makes them candidates as actuators in applications, such as changing the camber of a helicopter blade, which require large strains or displacements. The modeling of these materials is difficult, however, due to both the large strains involved and the inherently nonlinear constitutive behavior of the materials. As detailed in Section 4, this has promoted the development of both energy-based and phenomenological models which can be inverted for linear control design.

The design of smart structures which utilize these materials requires both the characterization of their constitutive properties and the development of coupled models which quantify their interaction with underlying systems. Control laws must be compatible with the properties of the sensors and actuators as well as the mechanisms through which they interact with the system. For example, the previously mentioned actuators yield unbounded (discontinuous) input operators in the mathematical formulation of the control problem. The extension of control theories to this regime has been been completed in certain applications but is lacking in general. Moreover, all of the active materials exhibit nonlinear dynamics and hysteresis at high drive levels. This must be incorporated in both the models and control methods before the materials can be utilized to their full capability in smart structure design. While aspects of the nonlinear design have been addressed, the state of the theory lags far behind that for the linear case.

Because the dynamics of a smart structure are dependent upon the attributes of the constituent active materials, it is necessary to consider the development of linear, nonlinear and hysteretic constitutive relations, their incorporation in coupled system models, and subsequent control design in a concerted manner. This facilitates the development of models amenable to control design, and the formulation of control laws which are compatible with the physical attributes of the active sensor and actuator materials. Subsequent discussion of the active materials and their utilization in smart structures follows this philosophy. For each compound, aspects of the material behavior which are pertinent to model development are discussed and appropriate constitutive relations are presented. Finally, a variety of control techniques compatible with the linear and nonlinear models are outlined. While this provides a synopsis of only certain facets of model development and control design for smart structures, it illustrates issues which must be addressed in order to utilize the full potential of these systems.

\section{Piezoelectric and Electrostrictive Materials}

Smart structure technology utilizing piezoelectric and electrostrictive materials has evolved from solely passive devices for reducing structural vibrations to highly integrated systems which provide the capability for both sensing and actuating, adaptively modifying system dynamics and monitoring the health of the system. To illustrate facets of this technology and the control issues which it has raised, we first outline some prototypical applications employing piezoelectric and electrostrictive transducers. A crucial aspect of control design is the development of appropriate models and this forms the focus of much of this section. Representative examples of current control designs and directions for future research are then summarized in Section 2.5.

\subsection{Piezoelectric and Electrostrictive Applications}

\subsubsection{Structural Applications}

Many of the initial applications utilizing both the sensing and actuating capabilities of piezoelectric materials focussed on vibration attenuation or the reduction of structure-borne noise in structural acoustic systems. To illustrate the first application, consider the thin beam with surface-mounted piezoceramic patches depicted in Figure 1. Through the application of diametrically out-of-phase voltages to the 

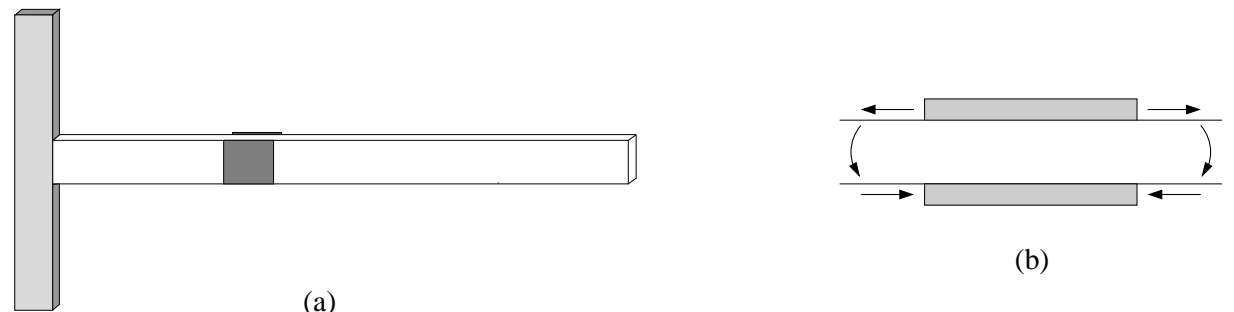

(b)

(a)

Figure 1: (a) Thin beam with surface-mounted piezoceramic patches. (b) Bending moments generated by out-of-phase voltages to the patches.

patches, bending moments are created in the beam which produce transverse displacements. This provides the actuator capabilities for the configuration. The sensor capabilities are provided by the direct piezoelectric effect in which stresses in the patch produce charges and corresponding voltages. A truly integrated smart structure utilizes either self-sensing actuators [27] or sensor-actuator pairs to provide the observations and inputs required for vibration attenuation.

While all piezoelectric materials exhibit a certain degree of hysteresis, it is minimal at low drive levels and can in certain applications be reduced by charge or current control [58]. Moreover, the feedback mechanisms employed in many control applications can further reduce the hysteresis exhibited by the materials. Hence the majority of structural control applications utilizing piezoceramic transducers have relied on linear models and linear control methods. The assumption of linearity in piezoelectric materials fails, however, at high drive levels or high accuracy applications such as micropositioning [34, 35]. In such regimes, hysteresis must be accommodated in both the models and control designs. In Section 2.3 we outline two hysteresis models which are amenable to inversion and hence facilitate the construction of an inverse compensator for linear control design.

Relaxor ferroelectric materials such as the electrostrictive PMN-PT-BT provide the capability for generating large strains with minimal hysteresis if employed in the diffuse transition region. As detailed in Section 2.2, however, the relation between input fields and generated strains is nonlinear at both low drive levels and near saturation. While this must be accommodated in control design, it also provides the possibility for designing adaptive structures in which coupling constants and sensitivities can be tuned by prescribing a bias field commensurate with a specific region of the nonlinear curve [63].

The dual sensor and actuator capabilities of piezoelectric and electrostrictive materials also provide the possibility for health monitoring in smart structures. As detailed in [9], this can be accomplished by driving the structure using the actuator facility of the material and sensing its response using the direct piezoelectric or electrostrictive effects. The status of the structure can then be evaluated using various criteria. The simplest strategy is to compare the response to baseline data for the original structure. If significant deviations are detected, more detailed analysis can be performed. If sufficiently accurate models are employed, this can include the identification of defects through least squares fits to the data. Details regarding these procedures are provided in [9] and included references.

In addition to vibration control and health monitoring, piezoelectric and electrostrictive actuators are increasingly employed to provide shape or structural modifications which enhance system performance. A prototypical example from [49] is the flap assembly depicted in Figure 2. In this design, the strains produced by stacked piezoelectric actuators are amplified by a rod and cusp assembly to provide the stroke required to rotate flaps. This research is directed at modifying the aerodynamic properties of aircraft wings or helicopter rotor blades. 

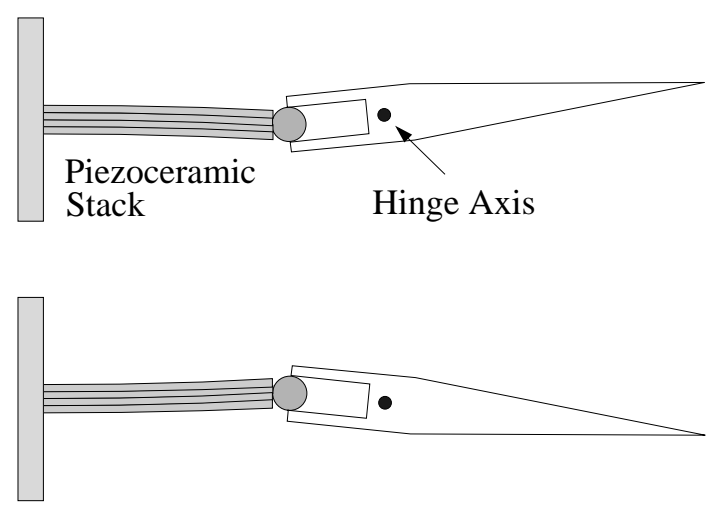

Figure 2: Piezoceramic stack used for flap rotation (after [49]).

\subsubsection{Structural Acoustic Applications}

A second class of smart structures in which piezoceramic actuators and sensors have played a fundamental role are those involved in structural acoustic systems. Structure-borne noise arises in settings ranging from aircraft and automobiles to fields emanating from high voltage transformers. In all cases, the unwanted noise is generated by structural vibrations produced by an adjacent source (e.g., aircraft engines, impinging flowfields, vibrating machinery, electromagnetic cores). Smart materials provide the capability for reducing structure-borne noise by modifying the structural dynamics to regimes which couple less effectively with acoustic fields as depicted in Figure 3. This capability can be enhanced through the use of models which predict sound power levels as a function of structural displacements and velocities. In this case, piezoceramic patches can be utilized to sense the structural strains and produce bending moments in accordance with a structural acoustic model-based control law [19].

The sound pressure levels encounted in originally considered aircraft, automotive and industrial systems were typically linear, and linear PZT models were employed for characterization and control design. As noted in Section 2.5, feedforward methods are currently employed for many structural acoustic applications. Because these techniques rely on superposition principles, they are inherently linear and hence require either linear models or linear filtering techniques such as inverse compensation.

Current and future structural acoustic applications are beginning to focus on regimes which are highly nonlinear and will require the development of nonlinear models and control techniques. For example, the sound pressure levels produced in a space launch vehicle payload fairing during liftoff or

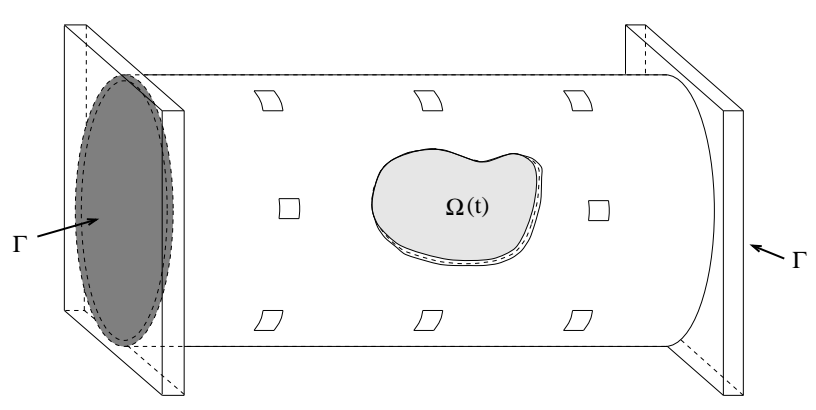

Figure 3: Thin shell with surface-mounted piezoceramic patches which encloses and acoustic cavity. 
impinging on an aircraft bay at supersonic speeds can exceed $150 \mathrm{~dB}$ [29, 72, 91]. Smart materials employed in these applications will quite likely be operating in highly nonlinear ranges which will necessitate the use of nonlinear constitutive and hysteresis models of the type illustrated in Section 2.3.

\subsubsection{High Performance Transducers}

An area of research which has the potential for significantly affecting smart structure design is the development of high performance transducers which employ piezoelectric or electrostrictive materials. Because these transducers can exhibit nonlinear dynamics and hysteresis, their incorporation will also necessitate the use of nonlinear models of the type outlined in Section 2.3 and control methods commensurate with these models.

The number of new high performance transducers that have recently been developed is vast so we restrict our attention to three types and refer the reader to [63] for general discussion concerning the state of the art in this area.

The first are THUNDER actuators which are typically comprised of a piezoceramic wafer, a metallic backing material, hot melt adhesive layers, and optional metallic top layers $[39,60]$. The manufacturing process produces a characteristic curved shape and provides the actuators with the robustness necessary to withstand the high voltages required for large force and strain generation. Because the actuators operate at extremely high drive levels, the relation between the input field and generated strains exhibits significant hysteresis and nonlinear constitutive relations which must be accommodated in models and subsequent control design.

A second class of composite transducers are flextensional designs which utilize PZT or PMN-PT-BT drivers $[21,36]$. Prototypical designs are depicted in Figure 4, and the reader is referred to Section 3.1 and [24] for discussion about analogous transducers which employ magnetostrictive drivers. This class of transducers was originally developed for sonar projection but due to its wide range of hydrodynamic response, it has recently been used for applications including oil exploration and underwater imaging. For high drive levels and electrostrictive drivers, nonlinear models must be employed to characterize the drive dynamics. A second area of modeling and optimal design involves the determination of cap geometries which produce desired frequency responses for both sending and receiving hydrostatic signals. Because the transducers have both tunable actuator and sensor attributes, they provide a unique capability for certain adaptive smart structure applications.

The final class of actuators which we mention are the inch worm actuators which utilize piezoelectric, electrostrictive or magnetostrictive materials as drive elements and clamps [24]. A hybrid design utilizing piezoceramic and magnetostrictive components is illustrated in Figure 21 of Section 3.1. Such designs

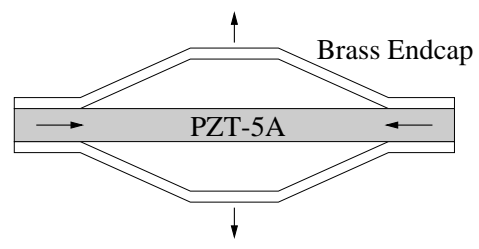

(a)

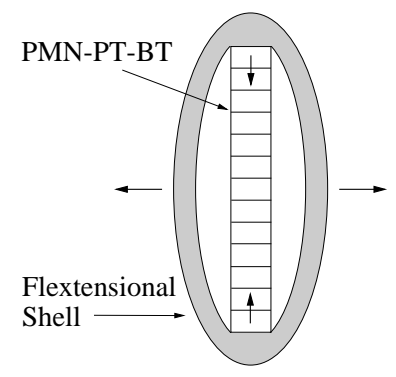

(b)

Figure 4: Flextensional piezoceramic and electrostrictive transducers. (a) Piezoceramic cymbal actuator (after [21]) and (b) PMN- PT-BT transducer (after [36]). 
provide the capability for essentially unlimited displacements with speeds currently on the order of $1 \mathrm{~mm} / \mathrm{sec}$. As the designs and drive electronics improve, the utilization of such devices in smart structure applications should continue to increase.

\subsection{Physical Properties of the Materials}

To motivate the models currently employed for smart structures employing ferroelectric and relaxor ferroelectric materials, it is necessary to understand certain characteristics of the material properties. For example, the ionic biases produced during the poling of piezoelectric materials are the source of bidirectional strains and quasilinear behavior at low drive levels whereas the hysteresis is a direct consequence of the domain structure inherent to ferroelectric materials. In this section, we summarize the material properties pertinent to subsequent model development and control design. We consider the commonly employed piezoelectric material $\mathrm{Pb}(\mathrm{Zr}, \mathrm{Ti}) \mathrm{O}_{3}$ and the electrostrictive material $\mathrm{Pb}(\mathrm{Mg}, \mathrm{Nb}) \mathrm{O}_{3}$ which are respectively referred to as PZT and PMN. For brevity, we focus on the ferroelectric properties of these materials and refer the reader to [37] for details concerning the physical behavior of relaxor ferroelectrics when employed in the diffuse transition region.

\subsubsection{Ferroelectric Domains}

Currently employed PZT transducer materials are comprised of $\mathrm{PbTi}_{1-x} \mathrm{O}_{3}$ and $\mathrm{PbZr}_{x} \mathrm{O}_{3}$ compounds with $x$ chosen to optimize electromechanical coupling. To simplify this discussion, we focus solely on $\mathrm{PbTi}_{1-x} \mathrm{O}_{3}$ and refer the reader to [63] for a general discussion of piezoelectric material properties.

As illustrated in Figure 5, the material distorts from a cubic to tetragonal form as it is cooled below the Curie point. This is manifested in the biasing of the $\mathrm{Ti}^{4+}$ ion toward an $\mathrm{O}^{2-}$ pair to minimize the

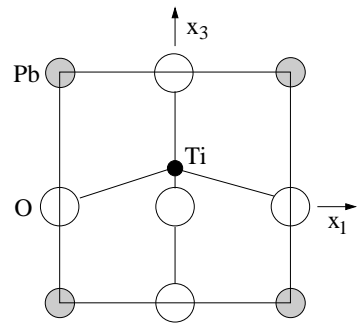

(a)

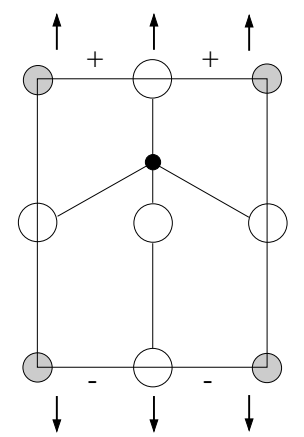

(c)

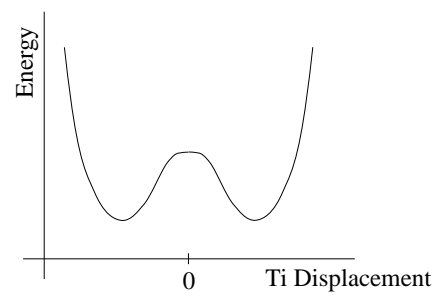

(b)

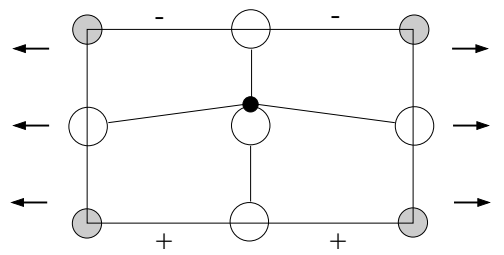

(d)

Figure 5: (a) Polar form of $\mathrm{PbTiO}_{3}$; (b) Potential energy as a function of Ti position along the $x_{3}$ axis; (c) Change in polarization $\Delta P_{3}=d_{33} \sigma_{3}$ due to an applied transverse stress $\sigma_{3}$; (d) Change in polarization $\Delta P_{3}=d_{31} \sigma_{1}$ due to an axial stress $\sigma_{1}$ (after [63]). 


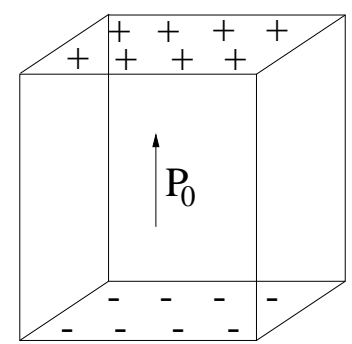

(a)

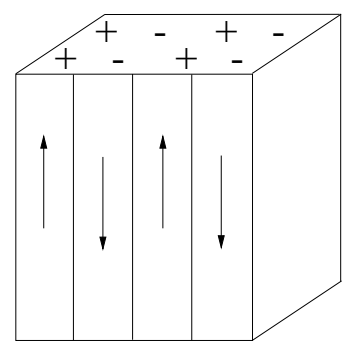

(b)

Figure 6: (a) Surface charge due to a spontaneous polarization. (b) twinned $180^{\circ}$ domains which form to minimize electrostatic energy.

potential energy and it produces a spontaneous polarization within the cell. As a direct consequence of the formation of spontaneous polarization is the generation of a surface charge and depolarizing field as depicted in Figure 6. The minization of electrostatic energy then produces a twinned $180^{\circ}$ domain structure where domains refer to regions where dipoles moments are highly aligned and domain walls denote transition regions between domains. Furthermore, the generation of stresses in the material as it cools produces $90^{\circ}$ domains which minimize strains and the corresponding elastic energy. We note that while polarization models must accommodate the switching of both $90^{\circ}$ and $180^{\circ}$ domains, the former is of primary interest when quantifying the strains generated by the material.

The discussion thus far pertains to single crystals whereas the piezoelectric materials used in smart structure applications are polychrystalline and, due to the random orientation of grains and domains, have no net polarization. To produce the electrical anisotropy which provides piezoelectric compounds with sensor and actuator capabilities, it is necessary to pole the material by applying an intense DC field at elevated temperatures slightly below the Curie point. This partially aligns domains which produces a net polarization in the material.

Two manifestations of the piezoelectric effect for poled ferroelectric materials are utilized in smart structure applications. The direct effect involves the generation of a charge when stress is applied to the material and provides the material with its sensor capabilities. The converse effect reflects the strain generated by an applied field and is used for actuator applications. As illustrated in Figure 5, the applications of a stress $\sigma_{3}$ parallel to the dipole produces an enhancement of the spontaneous polarization whereas the application of a perpendicular stress $\sigma_{1}$ decreases the spontaneous polarization. Hence the piezoelectric coupling coefficient $d_{33}$ is positive while $d_{31}$ is negative.

\subsubsection{Hysteresis}

An important manifestation of the ferroelectric nature of piezoelectric and electrostrictive materials is the inherent hysteresis observed at moderate to high input levels. A typical hysteresis relation between the input field $E$ and polarization $P$ for PZT5A is plotted in Figure 7 . The nearly vertical burst region of the curve is attributed to $180^{\circ}$ domain nucleation and growth. Near saturation, the material acts as a single domain and subsequent changes in polarization are due to the extension of dipoles through ion displacement.

Sigmoidal hysteresis of the form depicted in Figure 7 is typically attributed to the restriction of domain wall movement by inclusions or pinning sites inherent to the material. These sites can be caused by impurities, second-phase materials, or stress nonhomogeneities which in many cases are not only unavoidable but are necessary for the material's performance. The pinning of domain walls at these 


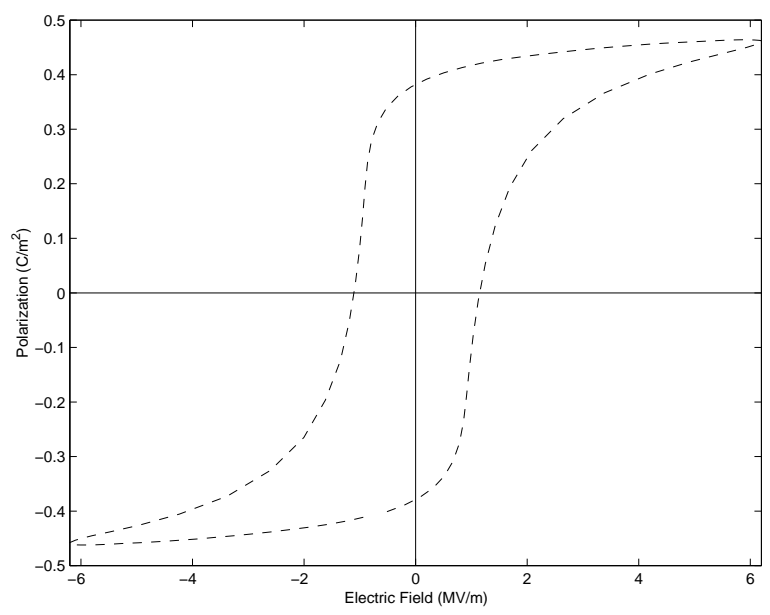

Figure 7: Hysteresis observed in a PZT5A wafer in response to a $1600 \mathrm{~V}$ input.

locations results from the reduction in electrostatic energy which occurs at the inclusions. A complete discussion of the mechanisms which underlie domain wall pinning and movement is beyond the scope of this presentation and we refer the reader to [80] for details and references concerning these phenomena.

For subsequent model development, we simply note that the motion of domain walls pinned at inclusions is comprised of two primary components: bending and translation. Bending occurs at low input field levels before domain walls have attained sufficient energy to move out of local energy wells $[15,30,54]$. This motion is reversible and accounts for a large percentage of the polarization change in hard PZT materials. At higher field levels, the local energy barriers are overcome and domain walls translate to a remote pinning site [15]. This provides an irreversible component to the polarization which is present in both hard and soft materials but plays a more major role in soft materials where lower energies are required to rotate domains. These reversible and irreversible components to the magnetization will be quantified in Section 2.3 to provide a hysteresis model for ferroelectric materials.

\subsection{Material Models}

To characterize the relations between applied fields and the polarization which results in ferroelectric and relaxor ferroelectric materials, we consider three classes of models. The linear models are applicable for piezoelectric materials at low drive levels where hysteresis and saturation phenomena are minimal. The second class of models includes higher order representations which are suitable for relaxor ferroelectric operating in the diffuse transition region where hysteresis is minimal. Finally, we outline two methods for quantifying the hysteresis observed in piezoelectric materials at high drive levels and electrostrictive materials operating in the ferroelectric range. The first hysteresis model is based on electrostrictive domain principles while the second is a Preisach approach which is phenomenological in nature. Both techniques are amenable to inversion which facilitates linear control design through the construction of an inverse compensator.

\subsubsection{Linear Model}

At low input field levels, piezoelectric materials exhibit bidirectional strains and nearly linear relations between the field, voltage, or polarization and the resulting strain. Similarly, the electric fields generated by the direct piezoelectric effect exhibit a nearly linear dependence upon the applied stress. The 
development of linear models relating the dielectric and elastic behavior of piezoelectric materials is attributed to Voigt, and the resulting coupled models are used for smart material characterization in numerous applications which require low input fields or voltages.

As detailed in $[61,64]$, the assumption of linear dielectric and elastic behavior respectively yields the relations

$$
\begin{gathered}
D_{i}=d_{i j k} \sigma_{j k}+\epsilon_{i j}^{\sigma} E_{j} \\
e_{i j}=s_{i j k \ell}^{E} \sigma_{k \ell}+d_{k i j} E_{k}
\end{gathered}
$$

as models for the direct and converse piezoelectric effects. Note that the Einstein summation convention is employed in both relations so that terms with repeated indices are summed. Here $D$ and $E$ denote the usual electric fields, $\sigma$ and $e$ respectively denote the applied stress and resulting strain, $\epsilon_{i j}^{\sigma}$ denotes components of the dielectric permittivity tensor measured at constant stress, and $s^{E}$ is the elastic compliance tensor measured at constant electric field. The first term on right side of the converse relation is due to Hooke's law while the second describes the strains which occur in a material which is free to deform in the absence of stresses. Finally, we note that the relation $D_{i}=\epsilon_{i j}^{\sigma} E_{j}$ in the direct model should be used with caution for ferroelectric materials since the permittivity is not only nonlinear but is a multi-valued map at high input levels.

The tensor formulations in (1) provide a complete description of the material behavior for general piezoelectric compounds. The number of coefficients can be reduced significantly, however, by invoking elastic symmetries and electric symmetries due to poling. When combined with a change of index, this provides a matrix system suitable for typical smart material applications.

We first note that for linear elastic materials, the stress and strain tensors are symmetric so that $\sigma_{i j}=\sigma_{j i}$ and $e_{i j}=e_{j i}$. The symmetry in stress and strain implies that $d_{i j k}$ is symmetric in $j$ and $k$ thus leaving 18 independent coefficients.

To formulate the system as a matrix equation, the indices $j k$ are replaced by a single index $m$ according to the following convention:

$$
\begin{array}{ccccccc}
11 & 22 & 33 & 23,32 & 31,13 & 12,21 & \text { Tensor } \\
1 & 2 & 3 & 4 & 5 & 6 & \text { Matrix }
\end{array}
$$

The coefficients $d_{i j k}$ are then written as $d_{i j k}=d_{i m}$ when $m=1,2,3$ and $d_{i j k}=\frac{1}{2} d_{i m}$ when $m=4,5,6$. The factor of $1 / 2$ accommodates the symmetry in $j$ and $k$. A similar convention is used to formulate the stress, strain and compliance tensors as matrices (e.g., $[23,71]$ ).

The number of coefficients $d_{i m}$ and stress-strain elements of interest are further reduced when we consider a material which is poled in the $x_{3}$ direction. As noted in [23], conical symmetry dictates that in this case, all of the piezoelectric coefficients are zero except $d_{31}=d_{32}, d_{33}$ and $d_{15}=d_{24}$.

The utilization of the structural and electrical symmetries along with matrix re-indexing then yields the system

$$
\left[\begin{array}{c}
e_{1} \\
e_{2} \\
e_{3} \\
e_{4} \\
e_{5} \\
e_{6} \\
\hline D_{1} \\
D_{2} \\
D_{3}
\end{array}\right]=\left[\begin{array}{cccccc|ccc}
s_{11}^{E} & s_{12}^{E} & s_{13}^{E} & 0 & 0 & 0 & 0 & 0 & d_{31} \\
s_{12}^{E} & s_{11}^{E} & s_{13}^{E} & 0 & 0 & 0 & 0 & 0 & d_{31} \\
s_{13}^{E} & s_{13}^{E} & s_{33}^{E} & 0 & 0 & 0 & 0 & 0 & d_{33} \\
0 & 0 & 0 & s_{44}^{E} & 0 & 0 & 0 & d_{15} & 0 \\
0 & 0 & 0 & 0 & s_{44}^{E} & 0 & d_{15} & 0 & 0 \\
0 & 0 & 0 & 0 & 0 & s_{66}^{E} & 0 & 0 & 0 \\
\hline 0 & 0 & 0 & 0 & d_{15} & 0 & \epsilon_{11}^{\sigma} & 0 & 0 \\
0 & 0 & 0 & d_{15} & 0 & 0 & 0 & \epsilon_{11}^{\sigma} & 0 \\
d_{31} & d_{31} & d_{33} & 0 & 0 & 0 & 0 & 0 & \epsilon_{33}^{\sigma}
\end{array}\right]\left[\begin{array}{c}
\sigma_{1} \\
\sigma_{2} \\
\sigma_{3} \\
\sigma_{4} \\
\sigma_{5} \\
\sigma_{6} \\
\hline E_{1} \\
E_{2} \\
E_{3}
\end{array}\right]
$$


summarizing the converse and direct piezoelectric effects. In matrix form, the components can be written as

$$
\begin{aligned}
& D=d \sigma+\epsilon^{\sigma} E \\
& e=s^{E} \sigma+d^{T} E .
\end{aligned}
$$

Finally, by utilizing the relation $P_{i}=\epsilon_{0} \chi_{i j} E_{j}$, where $\chi_{i j}$ is the dielectric susceptibility tensor and $\epsilon_{0}$ is the permittivity of a vacuum, one can also express the strain in terms of the polarization through the relation

$$
e_{i j}=s_{i j k \ell}^{E} \sigma_{k \ell}+g_{\ell j k} \epsilon_{i \ell}^{\sigma} P_{k} \text {. }
$$

This formulation proves advantageous when modeling ferroelectric hysteresis in the relation between the field and polarization.

\subsubsection{Higher Order Anhysteretic Models}

The linear models (1)-(3) are valid only for poled materials with small to moderate applied stresses or fields. For general relaxor ferroelectrics and higher input levels, quadratic electrostrictive effects, saturation phenomena and hysteresis are significant and must be incorporated in the models. We summarize here higher order models for hysteresis-free (anhysteretic) behavior and address hysteresis in the next section.

When formulated in terms of the polarization, the converse and direct effects in centrosymmetric materials (e.g., electrostrictive materials) can be modeled by the relations

$$
\begin{aligned}
& E_{i}=-2 Q_{k \ell i j} \sigma_{k \ell} P_{j}+\mathcal{F}\left(P_{i}, T\right) \\
& e_{i j}=s_{i j k \ell}^{E} \sigma_{k \ell}+Q_{i j k \ell} P_{k} P_{\ell} .
\end{aligned}
$$

Here $Q$ is a fourth rank tensor of electrostrictive coefficients while $\mathcal{F}\left(P_{i}, T\right)$ is a nonlinear function of the polarization and temperature $T$ which incorporates the low field quadratic behavior as well as the saturation effects which occur at high drive levels.

To illustrate the derivation of appropriate functions $\mathcal{F}(P, T)$ and to provide the framework required for subsequent hysteresis models, we consider the modeling of hysteresis free (anhysteretic) PMN through the application of Boltzmann statistics. As detailed in [79, 80], the potential energy for a dipole moment $\mathbf{p}_{0}$ in an electric field $\mathbf{E}$ is

$$
\mathcal{E}=-\mathbf{p}_{0} \cdot \mathbf{E}=-p_{0} E \cos \theta
$$

where $p_{0}=\left|\mathbf{p}_{0}\right|, E=|\mathbf{E}|$. The balancing of thermal and electrostatic energy then yields the probability

$$
\mu(\mathcal{E})=C e^{-\mathcal{E} / k_{B} T}
$$

that a dipole occupies the energy state $\mathcal{E}$. Here $k_{B}$ denotes Boltzmann's constant and $C$ is a constant which is specified in a manner which ensures that integration over all possible configurations yields the total number of moments per unit volume. The assumption that the orientation of cells can be in any direction yields the classical Langevin relation

$$
P_{a n}=P_{s}\left[\operatorname{coth}\left(\frac{E_{e}}{a}\right)-\left(\frac{a}{E_{e}}\right)\right]
$$


where $P_{s}$ is the saturation polarization and

$$
E_{e}=E+\alpha P+2 Q_{33} P \sigma
$$

denotes the effective field acting at the domain level. For constant temperature, the parameters $\alpha$ and $a$ are given by $\alpha=\widehat{E} / P_{s}$ and $a=\widehat{E} T /\left(3 T_{c}\right)$ where $\widehat{E}$ denotes a scaling electric field and $T_{c}$ is the Curie temperature. A second model which results from the assumption that dipoles align only in the direction of the field or opposite to it is the Ising spin relation

$$
P_{a n}=P_{s} \tanh \left(\frac{E_{e}}{a}\right)
$$

where $a=\widehat{E} T / T_{c}$. The behavior of the two models is illustrated in Figure 8. We also refer the reader to [67] for an alternative approach to modeling the anhysteretic polarization.

To obtain a constitutive relation commensurate with (4), the characterization must be polarization based. The necessary inversion can be accomplished for the Ising spin model which yields

$$
E=-2 Q_{33} P \sigma-\alpha P+\frac{\widehat{E} T}{T_{c}} \operatorname{arctanh} \frac{P}{P_{s}} .
$$

Hence for this model, the function $\mathcal{F}$ in (4) is given by

$$
\mathcal{F}(T, P)=-\alpha P+\frac{\widehat{E} T}{T_{c}} \operatorname{arctanh} \frac{P}{P_{s}} .
$$

Further details concerning the derivation and attributes of this model are provided in $[79,80]$.

\subsubsection{Hysteresis Models - Domain Wall Model}

As detailed in Section 2.2, hysteresis in the relation between the applied field and resulting polarization is an inherent property of all ferroelectric materials. We summarize here two techniques for modeling hysteresis in high drive level applications. The first is based on domain energy relations while the second illustrates a phenomenological Preisach characterization.

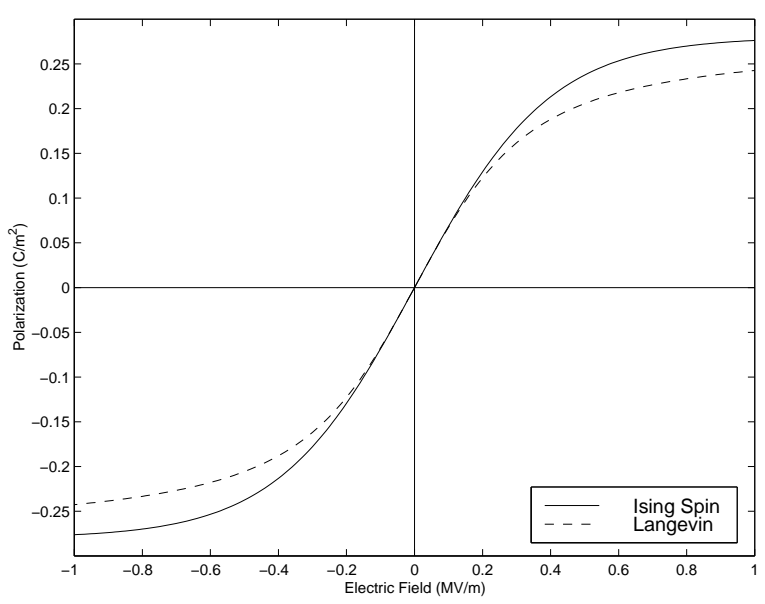

(a)

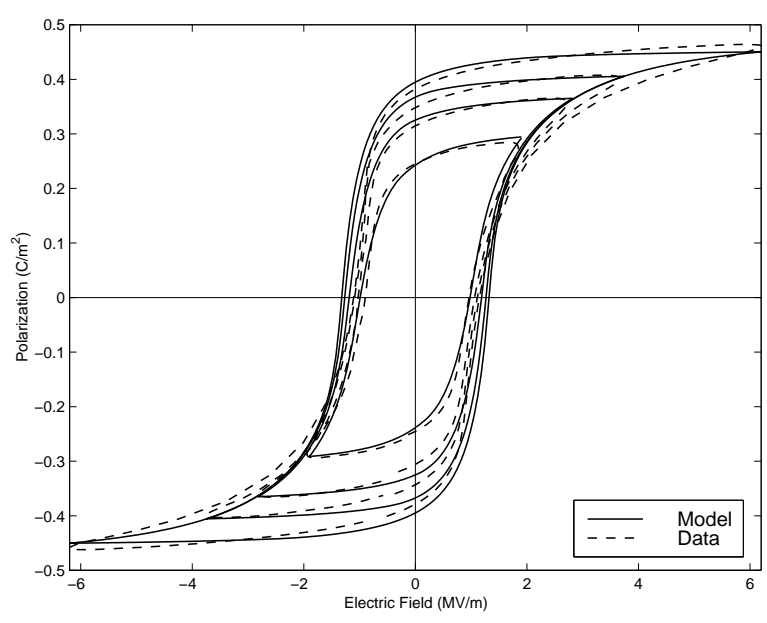

(b)

Figure 8: (a) Ising spin and Langevin models for the anhysteretic polarization; (b) Fit to $600 \mathrm{~V}, 800 \mathrm{~V}$, $1000 \mathrm{~V}$ and $1600 \mathrm{~V}$ PZT5A data using the model (14) with one set of parameters. 
The domain model is based on the observation that due to inclusions in the material, changes in polarization resulting from domain wall movement have irreversible and reversible components. To quantify the irreversible polarization, it is noted in $[79,80]$ that the polarization level for a given effective field can be expressed as that for the ideal case minus losses required to break pinning sites. This yields the relation

$$
P_{i r r}=P_{a n}-k \frac{d P_{i r r}}{d E_{e}}
$$

where $P_{a n}$ is defined by (5) or (7) and $k$ quantifies the average energy required to break pinning sites. The formulation of (10) in terms of the applied field $E$ yields the differential equation

$$
\frac{d P_{i r r}}{d E}=\widetilde{\delta} \frac{P_{a n}-P_{i r r}}{k \delta-\alpha\left(P_{a n}-P_{i r r}\right)}
$$

where the parameter $\delta=\operatorname{sign}(d E)$ ensures that the energy required to break pinning sites always opposes changes in polarization. The inclusion of the parameter $\widetilde{\delta}$ given by

$$
\widetilde{\delta}=\left\{\begin{array}{l}
1,\left\{d E>0 \text { and } P<P_{a n}\right\} \text { or }\left\{d E<0 \text { and } P>P_{a n}\right\} \\
0, \text { otherwise }
\end{array}\right.
$$

enforces that solely reversible polarization changes take place when the field reversed at saturation until the anhysteretic polarization is reached.

The second component of the polarization is the reversible polarization which models the effects of domain wall bending. To first approximation, this is modeled by the relation

$$
P_{\text {rev }}=c\left(P_{a n}-P_{\text {irr }}\right)
$$

where $c$ is a parameter which must be estimated for the specific application (see $[79,80]$ ).

The total polarization is then given by

$$
P=P_{r e v}+P_{i r r} .
$$

To implement the model, the effective field for a given field and irreversible polarization level is computed using (6). This value is then employed in either (5) or (7) to compute the corresponding anhysteretic polarization. The subsequent irreversible polarization is determined by numerically integrating (11). The total polarization is then specified by (14).

The experimental validation of the model for PMN transducers operating in the ferroelectric range is illustrated in [79, 80] while validation for various PZT compounds is addressed in [81, 82]. The predictive capability of the model is illustrated in Figure $8 \mathrm{~b}$. The parameters $a, \alpha, c, k$ and $P_{s}$ were estimated through a least squares fit to $600 \mathrm{~V}$ data and the model, with the same parameters, was used to predict the polarization resulting from $800 \mathrm{~V}, 1000 \mathrm{~V}$ and $1600 \mathrm{~V}$ inputs. The formulation of the model in terms of electrostatic energy principles provides it with the capability for accurately predicting the polarization throughout the operational range given only specified input levels.

A second advantage of this model lies in the fact that it can be inverted through consideration of a complementary ODE. This permits the construction of an inverse compensator which can be employed as a filter before the actuator to facilitate linear control design. Details regarding the construction of the inverse are provided in Section 3.3 where the analogous magnetic model is outline. 


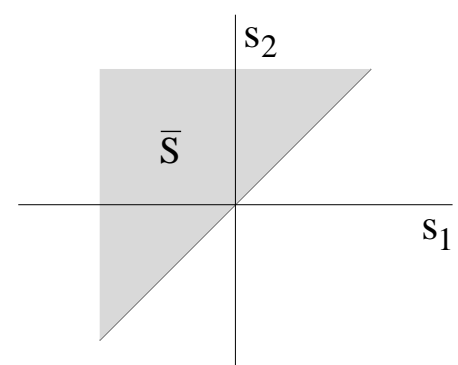

(a)

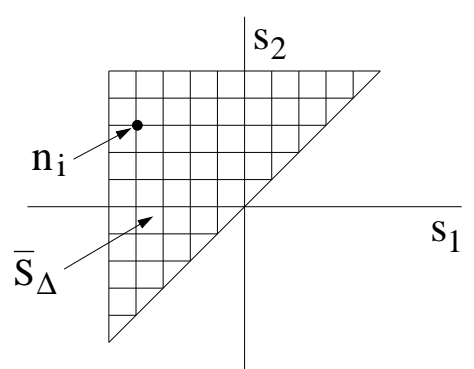

(b)

Figure 9: (a) Preisach plane $\bar{S}$ and (b) Restricted Preisach plane $\bar{S}_{\Delta}$.

\subsubsection{Hysteresis Models: Preisach Model}

A second technique for modeling hysteresis in a variety of smart material systems is based on the approximation of the multi-valued hysteresis map by a parallel collection of Preisach kernels. This provides a phenomenological or empirical characterization of the input/output relations which circumvents unmodeled or unknown physics. An advantage of this technique lies in its generality as evidenced by the fact that the same model can be used to characterize the hysteresis in piezoelectric materials or shape memory alloys (see Section 4.1). A second advantage is the fact that the resulting Preisach operator can be inverted to facilitate linear control design. A disadvantage of the approach is that it typically requires the estimation of a large number of nonphysical parameters and it does not utilize or provide physical insights regarding the material dynamics of the system.

We focus on the Krasnolselskii-Pokrovskii (KP) operator, also termed a generalized Preisach operator, since it provides continuity with respect to both time and parameters and is amenable to approximation $[6,7]$. The reader is referred to $[34,35]$ for a discussion of adaptive control design for classical Preisach operators defined in terms of piecewise constant kernels.

General Preisach operators are defined by

$$
\left[P_{\mu}(v, \xi)\right](t)=\int_{\bar{S}}\left[k_{s}(v, \xi(s))\right](t) d \mu(s)
$$

where $\mu \in \mathcal{M}$ is a finite signed Borel measure on the closed Preisach plane $\bar{S}=\left\{\left(s_{1}, s_{2}\right) \mid s_{1} \leq s_{2}\right\}$ as depicted in Figure 9. The input is denoted by $v$ while $\xi$ denotes initial states of the system. In classical Preisach formulations, the kernel $k_{s}$ is defined through a linear combination of piecewise constants whereas continuous, piecewise linear functions are employed in the KP kernel to ensure continuity with respect to time and parameters. The characterization of hysteresis using either kernel requires the identification of an appropriate measure $\mu(s)$ which acts as a weight on the kernel.

To define the KP kernel, we consider translates $r_{s_{1}}=r\left(v-s_{1}\right)$ and $r_{s_{2}}=r\left(v-s_{2}\right)$ of a Lipschitz continuous ridge function $r(v)$ as depicted in Figure 10. For time intervals $\left[t_{k-1}, t_{k}\right]$ where the input $v$ is monotone, a monotone operator is recursively defined by

$$
\left[\mathcal{R}\left(v, \mathcal{R}_{k-1}\right)\right](t)= \begin{cases}\max \left\{\mathcal{R}_{k-1}, r\left(v(t)-s_{2}\right)\right\} & \text { if } v \text { is non-decreasing } \\ \min \left\{\mathcal{R}_{k-1}, r\left(v(t)-s_{1}\right)\right\} & \text { if } v \text { is non-increasing }\end{cases}
$$

where

$$
\mathcal{R}_{k}=\left\{\begin{array}{l}
\mathcal{R}\left(v, \mathcal{R}_{k-1}\right)\left(t_{k}\right), \quad k=2, \cdots, j \\
\mathcal{R}_{0}=\xi, \quad k=1, \xi \in\{-1,1\}
\end{array}\right.
$$




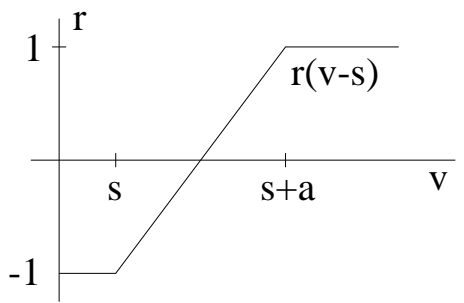

(a)

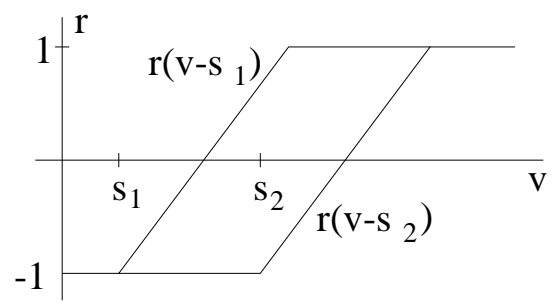

(b)

Figure 10: (a) Ridge function $r(v-s)$. (b) Hysteresis envelop provided by the translates $r_{1}$ and $r_{2}$ of the ridge function $r(v)$.

defines the values of $\mathcal{R}$ at times $t_{k}$. The KP kernel is then defined recursively on each subinterval by

$$
\left[k_{s}(v, \xi)\right](t)=\left[\mathcal{R}\left(v, \mathcal{R}_{k-1}\right)\right](t) \quad, \quad t \in\left[t_{k-1}, t_{k}\right] .
$$

A typical path for $k_{s}$ is depicted in Figure 11 while details concerning the properties of the kernel are provided in $[6,7]$.

To numerically implement the model, the measure $\mu(s)$ is approximated by the expansion

$$
\mu_{m}=\sum_{i=1}^{m} \alpha_{n_{i}} \delta_{n_{i}}
$$

where, as depicted in Figure 9, $n_{i}$ are nodes in the restricted Preisach plane $\bar{S}_{\Delta}=\left\{\left(s_{1}, s_{2}\right) \mid s_{\min } \leq\right.$ $\left.s_{1} \leq s_{2} \leq s_{\max }\right\}, \alpha_{n_{i}}$ are weights, and $\delta_{n_{i}}$ denotes the Dirac measure having an atom at $n_{i}$. The KP operator $P_{\mu}$ is then approximated by $P_{m}$ given by

$$
\left[P_{m}(v, \xi)\right](t)=\sum_{i=1}^{m}\left[k_{n_{i}}\left(v, \xi_{n_{i}}\right)\right](t) \alpha_{n_{i}} .
$$

As detailed in $[6,7,32]$, the continuity and convergence properties of the KP operator lead to wellposedness of the corresponding identification problem and provide a framework which is amenable to implementation.

An attribute of both the classical Preisach and KP operators, which is crucial to control design, is the fact that they can be inverted. We summarize here details regarding the inversion of the $\mathrm{KP}$ operator and refer the reader to Section 1.4 for an example illustrating its use in control design.

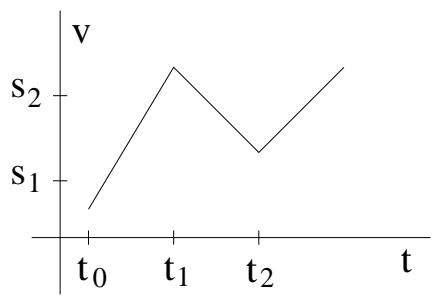

(a)

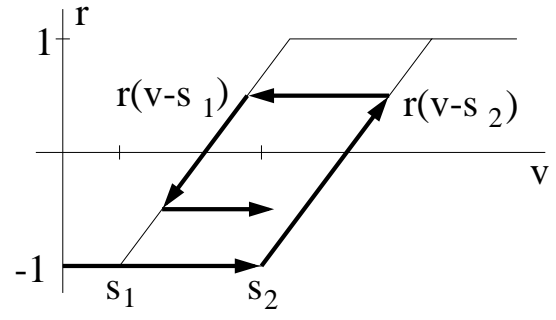

(b)

Figure 11: (a) Piecewise monotone input $v(t)$. (b) Output from the KP kernel in response to the input. 


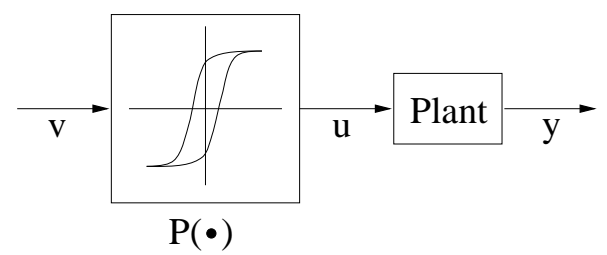

(a)

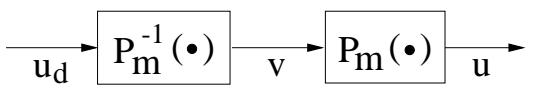

(b)

Figure 12: (a) Plant with input actuator hysteresis. (b) Approximate inverse compensator $P_{m}^{-1}$ for the hysteresis.

The subsequent development of the inverse operator $P_{m}^{-1}$ follows that in [32]. We first note that if $u_{d}(t)$ denotes the desired actuator input, the process of inverse compensation can be represented by

$$
u=P_{m}(v, \xi)=P_{m}\left(P_{m}^{-1}\left(u_{d}, \gamma\right), \xi\right)=u_{d}
$$

as illustrated in Figure 12.

With the definition $\Delta u_{d}=u_{d}(t)-u_{d}\left(t_{0}\right)$, it then follows that changes in the desired actuator input on the interval $\left[t_{0}, t_{1}\right]$ are given by

$$
\begin{aligned}
\Delta u_{d} & =P_{m}(v, \xi)-P_{m}\left(v_{0}, \xi\right) \\
& =\sum_{i=1}^{m}\left(\left[k_{n_{i}}\left(v, \xi_{n_{i}}\right)\right](t)-\left[k_{n_{i}}\left(v_{0}, \xi_{n_{i}}\right)\right](t)\right) \alpha_{n i} \\
& =\sum_{n_{i} \in \bar{S}_{\Delta}}\left[r\left(v-n_{i}\right)-r\left(v_{0}-n_{i}\right)\right] \alpha_{n_{i}} .
\end{aligned}
$$

For the piecewise linear ridge function

$$
r\left(v-s_{2}\right)=\left\{\begin{array}{cl}
1 & , s_{2} \leq v-a \\
-1+2\left(v-s_{2}\right) / a & , v-a<s_{2}<v \\
-1 & , v \leq s_{2}
\end{array}\right.
$$

depicted in Figure 10, the change in the kernel at each node $s_{2}=n_{i}$ is given by

$$
r\left(v-n_{i}\right)-r\left(v_{0}-n_{i}\right)=\left\{\begin{array}{cl}
0 & , n_{i} \leq v_{0}-a \\
\Delta r_{n_{i}} & , v_{0}-a<n_{i}<v \\
0 & , v \leq n_{i} .
\end{array}\right.
$$

It then follows that

$$
\Delta u_{d}=\sum_{n_{i} \in S^{+}} \alpha_{n_{i}}-\sum_{n_{i} \in S^{-}} \alpha_{n_{i}}+\sum_{n_{i} \in S^{\Delta}} \Delta r_{n_{i}} \alpha_{n_{i}}
$$

where

$$
\begin{aligned}
& S^{-}=\left\{n_{i} \in \bar{S}_{\Delta} \mid r\left(v-n_{i}\right)=-1\right\} \\
& S^{+}=\left\{n_{i} \in \bar{S}_{\Delta} \mid r\left(v-n_{i}\right)=1\right\} \\
& S^{\Delta}=\left\{n_{i} \in \bar{S}_{\Delta} \mid-1<r\left(v-n_{i}\right)<1\right\} .
\end{aligned}
$$

If $\Delta u$ is less than or equal to the distance between adjacent nodes $n_{i}$, then

$$
\Delta r_{i}=2\left(v-v_{0}\right) / a
$$


and (19) can be solved for $v$ to obtain the relation

$$
P_{m}^{-1}\left(u_{d}, \gamma\right)=v_{0}+a\left(\Delta u_{d}-\sum_{n_{i} \in S^{+}} \alpha_{n_{i}}+\sum_{n_{i} \in S^{-}} \alpha_{n_{i}}\right) /\left(2 \sum_{n_{i} \in S^{\Delta}} \alpha_{n_{i}}\right)
$$

for the inverse operator. As detailed in [32], an iterative procedure provides the inverse if $\Delta u$ is greater than the distance between nodes. Experimental validation of this modeling and inversion procedure for quantifying hysteresis for a piezoceramic stack is reported in [32].

\subsection{System Models}

The discussion in Section 2.1 indicates certain smart structure applications which utilize piezoceramic and electrostrictive actuators while the models of Section 2.3 characterize certain material properties for these actuator-sensor compounds. In this section, we illustrate the incorporation of the constitutive relations from Section 2.3 in prototypical structural and structural acoustic systems, and the formulation of the system model in a format suitable for control implementation. For brevity, we consider a thin beam model and a coupled 2-D model as prototypes for structural and structural acoustic systems. While both represent simplified scenarios, they provide the framework required for modeling more complex systems as a prelude to control design.

\subsubsection{Beam with Piezoceramic Actuators and Sensors}

Consider a cantilevered thin beam with a pair of surface-mounted piezoceramic actuators as depicted in Figure 13. For modeling purposes the beam is assumed to have length $\ell$, width $b$, and thickness $h$. The density, Young's modulus and Kelvin-Voigt damping coefficient for the beam and piezoceramic patches are respectively denoted by $\rho_{b}, E_{b}, c_{D_{b}}$ and $\rho_{p e}, E_{p e}, c_{D_{p e}}$. The transverse beam displacement is given by $w$ while $p(t, x)$ denotes an exogenous surface force to the beam. We note that for this configuration, the linear piezoelectric equations (2) reduce to

$$
\begin{aligned}
D_{3} & =d_{31} \sigma_{3}+\epsilon_{3} E_{3} & & \text { Direct } \\
e_{3} & =\frac{1}{E_{p e}} \sigma_{3}+d_{31} \frac{V}{h_{p e}} & & \text { Converse }
\end{aligned}
$$

where $V$ denotes the voltage to the patches and we have employed the relation $E=V / h_{p e}$ in the

converse relation.

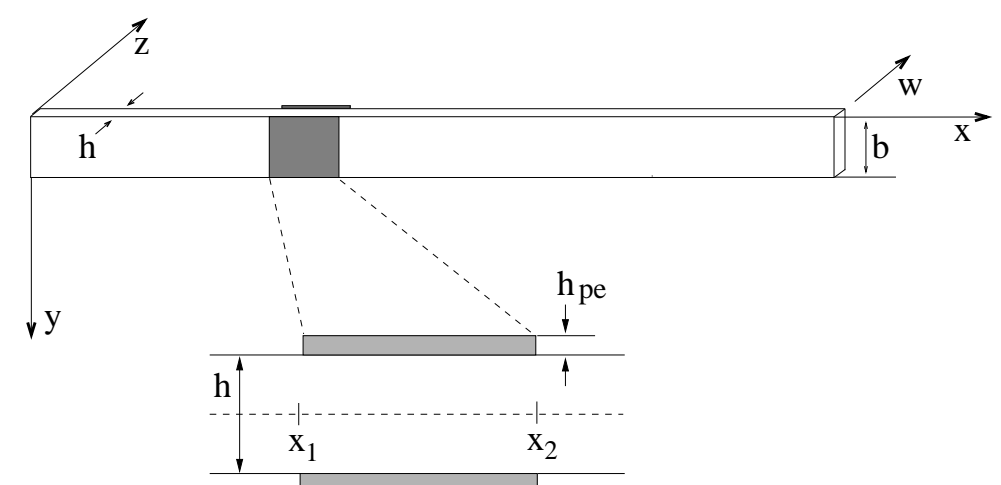

Figure 13: Thin beam with surface-mounted piezoceramic patches. 
As detailed in [9], force and moment balancing yields the strong form of the Euler-Bernoulli equations

$$
\begin{aligned}
& \rho(x) \frac{\partial^{2} w}{\partial t^{2}}(t, x)+\frac{\partial^{2} \mathcal{M}_{i n t}}{\partial x^{2}}(t, x)=p(t, x)+\frac{\partial^{2} \mathcal{M}_{\text {ext }}}{\partial x^{2}}(t, x) \\
& w(t, 0)=\frac{\partial w}{\partial x}(t, 0)=0 \\
& \mathcal{M}_{\text {int }}(t, \ell)=\frac{\partial \mathcal{M}_{\text {int }}}{\partial x}(t, \ell)=0
\end{aligned}
$$

along with appropriate initial conditions, as a model for the transverse beam dynamics. The composite density and internal bending moment are given by

$$
\begin{aligned}
& \rho(x)=\rho_{b} h b+2 \rho_{p e} b h_{p e} \chi_{p z t}(x) \\
& \mathcal{M}_{i n t}(t, x)=E I(x) \frac{\partial^{2} w}{\partial x^{2}}(t, x)+c_{D} I \frac{\partial^{3} w}{\partial x^{2} \partial t}(t, x)
\end{aligned}
$$

where the characteristic function $\chi_{p z t}$ delineates the location of the rods and

$$
\begin{aligned}
& E I(x)=\frac{E_{b} h^{3} b}{12}+\frac{2 b}{3} E_{p e} a_{3} \chi_{p z t}(x) \\
& c_{D} I(x)=\frac{c_{D_{b}} h^{3} b}{12}+\frac{2 b}{3} c_{D_{p e}} a_{3} \chi_{p z t}(x)
\end{aligned}
$$

with $a_{3}=\left(h / 2+h_{p e}\right)^{3}-h^{3} / 8$. The first term in the definition of $E I$ is the usual stiffness parameter for a uniform beam while the second is due to the passive material contributions

$$
2 \int_{h / 2}^{h / 2+h_{p e}} E_{p e} e_{3} b z d z=\frac{2 b}{3} E_{p e} a_{3}
$$

from the converse patch relation. The factor of two results from invoking the symmetry of the two patches. Integration of the strain rate for the beam and patch material yields the Kelvin-Voigt damping parameter.

For the case when the patches are driven diametrically out-of-phase, the integration of the free strain component of the converse relation yields the external moment

$$
\begin{aligned}
\mathcal{M}_{e x t}(t, x) & =2 \int_{h / 2}^{h / 2+h_{p e}} d_{31} E_{p e} b\left(V / h_{p e}\right) z d z \\
& =K^{B} \chi_{p z t} V(t)
\end{aligned}
$$

where $K^{B}=E_{p e} b d_{31}\left(h+h_{p e}\right)$ and $V(t)$ is the voltage to the patches. In applications, the actuator coefficient $K_{B}$ is typically identified for a given structure through a least squares fit to data.

To obtain a weak form of the model, we take the state to be the displacement $w$ in the state space $X=L^{2}(0, \ell)$ with the inner product

$$
\langle\phi, \psi\rangle_{X}=\int_{0}^{\ell} \rho \phi \psi d x .
$$

The space of test functions is taken to be $V=H_{L}^{2}(0, \ell) \equiv\left\{\phi \in H^{2}(0, \ell) \mid \phi(0)=\phi^{\prime}(0)=0\right\}$ with the inner product

$$
\langle\phi, \psi\rangle_{V}=\int_{0}^{\ell} E I \phi^{\prime \prime} \psi^{\prime \prime} d x .
$$


It should be noted that with these choices, $V$ is continuously and densely embedded in $X$. Hence one has the Gelfand triple $V \hookrightarrow X \simeq X^{*} \hookrightarrow V^{*}$ with the pivot space $X$.

A weak form of the model is then given by

$$
\int_{0}^{\ell} \rho \ddot{w} \psi d x+\int_{0}^{\ell} \mathcal{M}_{i n t} \psi^{\prime \prime} d x=\int_{0}^{\ell} \mathcal{M}_{e x t} \psi^{\prime \prime} d x+\int_{0}^{\ell} p \psi d x
$$

for all $\psi \in V$.

The equations (21) and (25) with moments given by (22) and (24) model the actuator contributions to the structure. The sensor attributes are modeled using the direct piezoelectric relation in (20). For a beam in which strains are solely due to bending, the stress at a point $\left(x, z_{c}\right)$, where $z_{c}=\left(h+h_{p e}\right) / 2$ is the distance from the neutral axis of the beam to the center of the patch, is approximated by

$$
\sigma_{3}(t, x)=z_{c} E_{p e} \frac{\partial^{2} w}{\partial x^{2}}
$$

In the absence of an applied field $\left(E_{3}=0\right)$, the direct piezoelectric relation then yields

$$
D_{3}=d_{31} z_{c} E_{p e} \frac{\partial^{2} w}{\partial x^{2}}
$$

The charge $q(t)$ developed on the patch as the material strains is computed by integrating (26) over the area of the patch to obtain

$$
\begin{aligned}
q(t) & =b \int_{x_{1}}^{x_{2}} d_{31} z_{c} E_{p e} \frac{\partial^{2} w}{\partial x^{2}} d x \\
& =E_{p e} d_{31} b z_{c}\left[\frac{\partial w}{\partial x}\left(t, x_{2}\right)-\frac{\partial w}{\partial x}\left(t, x_{1}\right)\right] .
\end{aligned}
$$

Finally, the voltage $V_{p}$ generated by the patch is computed by dividing the charge by the capacitance $C_{p e}$ to obtain

$$
V_{p}(t)=K^{s}\left[\frac{\partial w}{\partial x}\left(t, x_{2}\right)-\frac{\partial w}{\partial x}\left(t, x_{1}\right)\right]
$$

where

$$
K^{s}=\frac{E_{p e} d_{31} b\left(h+h_{p e}\right)}{2 C_{p e}} .
$$

Hence the patch can be used to sense accumulated strain.

The previous discussion illustrates the use of the direct and converse piezoelectric effects for employing piezoceramic patches as sensors and actuators in smart structures. Additionally, the dual piezoelectric properties provide the patches with the capability for simultaneous sensing and actuating. Self-sensing piezoceramic actuators were developed somewhat concurrently by Dosch et al. [27] and Anderson and Hagood [2] while adaptive sensoriactuators were developed by Cole and Clark to address difficulties associated with drifting capacitance [20]. Whether the patches are employed as isolated sensors and actuators or as collocated sensoriactuators, they provide significant potential as transducers in smart structures. 


\subsubsection{Finite Dimensional Model}

It is necessary to approximate the dynamics of the infinite-dimensional model (25) before it can be employed for either simulation purposes or control design. We employ a Galerkin approximation in the spatial variable to obtain a semidiscrete ODE system in time which is amenable to control formulation. Specifically, the spatial basis is taken to be $\left\{\phi_{j}\right\}_{j=1}^{m+1}$ where $\phi_{j}(x)$ denotes the $j^{\text {th }}$ cubic $B$-spline modified to satisfy the fixed left boundary condition [68]. Approximate solutions

$$
w^{m}(t, x)=\sum_{j=1}^{m+1} w_{j}(t) \phi_{j}(x)
$$

are then considered in the subpace $V^{m}=\operatorname{span}\left\{\phi_{j}\right\}$. To obtain a vector ODE system, the infinite dimensional system (25) is restricted to $V^{m}$ and posed in first-order form to yield

$$
\begin{aligned}
& \dot{x}(t)=A x(t)+B u(t)+g(t) \\
& x(0)=x_{0} .
\end{aligned}
$$

The state, control input, and control matrices are respectively defined by $x(t)=\left[w_{1}(t), \cdots, w_{m+1}(t)\right.$, $\left.\dot{w}_{1}(t), \cdots, \dot{w}_{m+1}(t)\right], u(t)=\left[u_{1}(t), \cdots, u_{s}(t)\right]$ and $B=K^{B} b$. The component system matrices have the form

$$
\begin{aligned}
& A=\left[\begin{array}{cc}
0 & I \\
\widetilde{Q}^{-1} K & \widetilde{Q}^{-1} C_{D}
\end{array}\right] \\
& b=\left[\begin{array}{c}
0 \\
\widetilde{Q}^{-1} \widetilde{b}
\end{array}\right] \\
& g(t)=\left[\begin{array}{c}
0 \\
\widetilde{Q}^{-1} p(t)
\end{array}\right]
\end{aligned}
$$

where

$$
\begin{array}{ll}
{[\widetilde{Q}]_{i j}=\int_{0}^{\ell} \rho \phi_{i} \phi_{j} d x} & {[\widetilde{b}]_{i}=\int_{m a g} \phi_{i}^{\prime \prime} d x} \\
{[K]_{i j}=\int_{0}^{\ell} E I \phi_{i}^{\prime \prime} \phi_{j}^{\prime \prime} d x} & {[p(t)]_{i}=\int_{0}^{\ell} p(t, x) \phi_{i} d x} \\
{\left[C_{D}\right]_{i j}=\int_{0}^{\ell} c_{D} I \phi_{i}^{\prime \prime} \phi_{j}^{\prime \prime} d x .} &
\end{array}
$$

Details regarding the construction of the finite dimensional system can be found in $[9,77]$.

\subsubsection{Nonlinear Actuator Models}

The linear system model (29) is based on the assumption that the linear constitutive relations (20) adequately model the actuator and sensor effects of the patches. As detailed in Section 2.2, however, both piezoceramic and electrostrictive materials exhibit nonlinear constitutive behavior and varying degrees of hysteresis at high drive levels which necessitates consideration of the hysteresis models discussed in 
Section 2.3. The incorporation of domain wall or Preisach hysteresis models with the beam equations yields the system

$$
\begin{aligned}
& \dot{x}(t)=A x(t)+[B(u)](t)+g(t) \\
& x(0)=x_{0}
\end{aligned}
$$

upon approximation. The nonlinear input $[B(u)](t)=[P(u)](t) b$ where $b$, given by $(30)$, incorporates the spatial location of the actuator and $[P(u)](t)$ quantifies the hysteresis and nonlinear behavior. Details regarding the construction of $B(u)$ for the analogous magnetic domain wall hysteresis model are provided in Section 3.5 while it construction for the Preisach operator is illustrated in the context of shape memory alloys in Section 4.1.

\subsubsection{Structural Acoustic System}

The second example which we consider is a prototypical structural acoustic system in which piezoceramic actuators mounted on the structure are employed as actuators and sensors. To simplify the discussion, we consider a rectangular cavity with a vibrating beam at one end as depicted in Figure 14 . The remaining cavity walls $\Gamma$ are assumed to reflect all acoustic vibrations. A force $\hat{p}$ modeling an exogenous pressure field drives the system and produces unwanted noise in the cavity $\Omega$.

As detailed in [9], for sound pressure levels on the order of those typically encountered in many aeronautical and automotive applications, the acoustic dynamics in a medium with density $\rho_{f}$ can be modeled by the wave equation

$$
\begin{aligned}
& \frac{\partial^{2} \phi}{\partial t^{2}}=c^{2} \Delta \phi \quad, \quad(x, y) \in \Omega \\
& \nabla \phi \cdot \hat{n}=0 \quad, \quad(x, y) \in \Gamma
\end{aligned}
$$

where $\phi$ denotes the acoustic potential, $c$ is the speed of sound, $p=\rho_{f} \frac{\partial \phi}{\partial t}$ is the pressure and $\nabla \phi=-u$ is the field velocity. The balancing of velocity at the beam surface $\left(\frac{\partial \phi}{\partial n}=w_{t}\right)$ and incorporation of

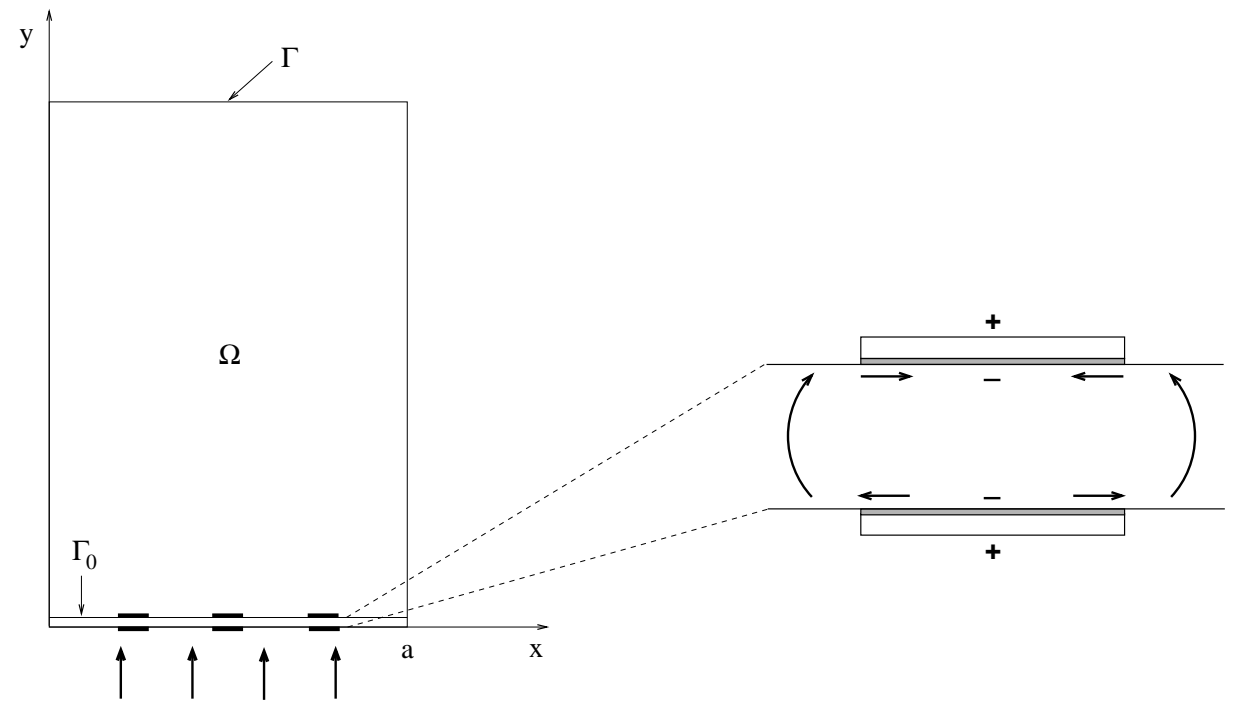

(a)

(b)

Figure 14: (a) Structural acoustic system; (b) Piezoceramic patches driven out-of-phase to create pure bending moments. 
pressure as a force on the beam then yields the coupled model

$$
\begin{aligned}
\int_{\Omega} & \frac{\rho_{f}}{c^{2}} \frac{\partial^{2} \phi}{\partial t^{2}} \xi d \omega+\int_{\Omega} \rho_{f} \nabla \phi \cdot \nabla \xi d \omega \\
& +\int_{\Gamma_{0}} \rho \frac{\partial^{2} w}{\partial t^{2}} \eta d \gamma+\int_{\Gamma_{0}} E I \frac{\partial^{2} w}{\partial x^{2}} \frac{\partial^{2} \eta}{\partial x^{2}} d \gamma+\int_{\Gamma_{0}} c_{D} I \frac{\partial^{3} w}{\partial x^{2} \partial t} \frac{\partial^{2} \eta}{\partial x^{2}} d \gamma \\
& +\int_{\Gamma_{0}} \rho_{f}\left(\frac{\partial \phi}{\partial t} \eta-\frac{\partial w}{\partial t} \xi\right) d \gamma \\
= & \int_{\Gamma_{0}} \sum_{i=1}^{s} \mathcal{K}_{i}^{B} u_{i}(t) \chi_{p e_{i}}(x) \frac{\partial^{2} \eta}{\partial x^{2}} d \gamma+\int_{\Gamma_{0}} \hat{p} \eta d \gamma
\end{aligned}
$$

for all $(\xi, \eta) \in V$. The space of test functions is $V=\bar{H}^{1}(\Omega) \times H_{0}^{2}\left(\Gamma_{0}\right)$ where $\bar{H}^{1}(\Omega)$ denotes the quotient space of $H^{1}(\Omega)$ over constant functions and $H_{0}^{2}\left(\Gamma_{0}\right)$ is the subset $H^{2}\left(\Gamma_{0}\right)$ whose elements satisfy the fixed boundary conditions for the beam. This model provides a baseline for comparison since it incorporates the full physics for the system.

A second technique used to model the acoustic field produced by the vibrating structure is to estimate the RMS sound power radiated from the structure through a discretization of Raleigh's integral. Radiation filters are then constructed to incorporate the physical mechanisms modeling the structural acoustic coupling. These filters are combined with a state variable model of the structure, which includes the input and output provided by piezoceramic actuators and sensors, to provide an augmented plant model. Details concerning this modeling approach for structural acoustic systems can be found in [19]. While the accuracy of the approximations to the Raleigh integrals degrades near boundaries, the method can lead to significantly lower-order systems than obtained through a full-order approximation of (33). Furthermore, the prediction of sound levels solely through consideration of the structural vibrations permits the elimination of acoustic sensors and promotes the use of smart material transducers such as piezoceramic sensoriactuators. Hence the smart structure capabilities provided by these materials can be utilized in structural acoustic applications.

\subsection{Control Applications}

\subsubsection{Infinite and Finite Dimensional Control Problems}

The problem of determining optimal voltages to patches employed as actuators in structural and structural acoustic systems leads to the formulation of infinite dimensional control problems with the models (25) or (33) as constraints. Due to the nature of the patch inputs and outputs, these control problems typically involve unbounded (discontinuous) input and output operators and are quite often formulated as boundary control problems. The analysis of the linear quadratic regulator (LQR) problems associated with these systems and the determination of convergence criteria for the resulting finite dimensional systems has resulted in the development of a fairly extensive theory for linear models. Corresponding infinite dimensional theory for control systems with nonlinear or hysteretic inputs is far less complete. A detailed discussion of the infinite dimensional LQR theory associated with smart structures is beyond the scope of this paper and the reader is referred to $[3,5,9,55]$ for details concerning the state of this theory.

In subsequent discussion, we focus on finite dimensional control systems of the form

$$
\begin{aligned}
\dot{x}(t) & =A x(t)+[B(u)](t)+g(t) \\
y(t) & =C x(t)
\end{aligned}
$$


where $x$ is a $N \times 1$ state vector, $y$ denotes observations in $\mathbb{R}^{P}$ and $C$ is a $P \times N$ observation matrix whose elements are specified by the form of the observations (e.g., see [9, page 232]). In this section, we focus on the linear case $[B(u)](t)=B u(t)$ where $B$ is an $N \times s$ matrix for structures with $s$ actuator pairs. We then consider the nonlinear case in Section 3.5 in the context of magnetostrictive actuators.

\subsubsection{Feedback Control Design}

We consider two cases for the exogenous force $g(t)$ : (i) $g(t) \equiv 0$ which can be used when controlling systems subject to stochastic or modeling uncertainties, and (ii) $g(0)=g(\tau)$ which arises when modeling periodic exogenous inputs. For the first case, the LQR problem concerns the determination of a control $u$ which minimizes

$$
J(u)=\int_{0}^{\infty}\{\langle Q x(t), x(t)\rangle+\langle R u(t), u(t)\rangle\} d t
$$

subject to

$$
\begin{aligned}
& \dot{x}(t)=A x(t)+B u(t) \\
& y(t)=C x(t) .
\end{aligned}
$$

The matrix $Q$ is chosen to satisfy kinetic and potential energy criteria and $R$ weights the voltage to the $s$ patch pairs [4]. While theoretically attractive, the minimization of (34) requires full state information which is typically not available in applications. This necessitates the consideration of a dynamic compensator which estimates or reconstructs states before computing a feedback control.

As detailed in $[8,9]$, the optimal full-order control is specified by

$$
u(t)=-K x_{c}(t)
$$

where the estimated state satisfies

$$
\begin{aligned}
& \dot{x}_{c}(t)=A x_{c}(t)+B u(t)+F\left[y(t)-C x_{c}(t)\right] \\
& x_{c}(0)=x_{c_{0}} .
\end{aligned}
$$

Here $K$ and $F$ denote the compensator and feedback gains, respectively. We note that $K$ and $F$ are chosen so that the reconstruction error $\left|y(t)-y_{c}(t)\right| \rightarrow 0$ as $t \rightarrow \infty$. Under usual observability and controllability hypotheses (see [52]), the optimal feedback and compensator gains are given by

$$
\begin{aligned}
& K=R^{-1} B^{T} \Pi \\
& F=P C^{T} \widehat{R}^{-1}
\end{aligned}
$$

where $\Pi$ and $P$ are unique nonnegative-definite solutions to the feedback (regulator) and compensator (observer) algebraic Riccati equations

$$
\begin{aligned}
& \Pi A+A^{T} \Pi-\Pi B R^{-1} B^{T} \Pi+Q=0 \\
& P A^{T}+A P-P C^{T} \widehat{R}^{-1} C P+\widehat{Q}=0,
\end{aligned}
$$

respectively. As was the case with the matrices $Q$ and $R$, the matrices $\widehat{Q}$ and $\widehat{R}$ are design criteria for the specific control application under consideration. 
For case (ii) in which $g$ is assumed periodic and $\tau$ is commensurate with all frequencies present in the exogenous signal, the optimal control is determined by minimizing

$$
J_{\tau}(u)=\frac{1}{2} \int_{0}^{\tau}\{\langle Q x(t), x(t)\rangle+\langle R u(t), u(t)\rangle\} d t
$$

subject to

$$
\begin{aligned}
& \dot{x}(t)=A x(t)+B u(t)+g(t) \quad, \quad x(0)=x(\tau) \\
& y(t)=C x(t) .
\end{aligned}
$$

The optimal control in this case is given by

$$
u(t)=-k x_{c}(t)+R^{-1} B^{T} z(t)
$$

where the reconstructed state is specified by the differential equation

$$
\begin{aligned}
& \dot{x}_{c}(t)=A x_{c}(t)+B u(t)+F\left[y(t)-C x_{c}(t)\right]+g(t) \\
& x_{c}(0)=x_{c}(\tau) .
\end{aligned}
$$

and the tracking variables are solutions to the adjoint equation

$$
\begin{aligned}
& \dot{z}(t)=-[A-B K]^{T} z(t)+\Pi g(t) \\
& z(0)=z(\tau) .
\end{aligned}
$$

The feedback and observer gains $K$ and $F$ are defined in (38) and $\Pi$ is the solution to the feedback Riccati equation in (39).

We refer the reader to $[8,9]$ for details concerning the original infinite dimensional control problem, criteria which guarantee the convergence of finite dimensional gains, and examples illustrating the experimental implementation of the control method for a circular plate with surface-mounted piezoceramic actuators.

\subsubsection{Adaptive Feedforward Control}

Currently employed adaptive feedforward algorithms are based on the representation of the plant and control as finite impulse response (FIR) filters whose coefficients are determined using LMS algorithms. This discussion provides only an overview of the methods and readers are referred to [19] for details concerning feedforward control design for smart or adaptive structures, [62] for additional analysis concerning acoustic systems, and [50,89] for general theory of adaptive filters and feedforward control design. As will be noted in this summary, feedforward control methods are based on superposition principles and hence are linear by nature. Thus they are restricted to linear systems or systems which have been suitably linearized.

Feedforward control design for structural or structural acoustic systems which employ piezoceramic sensors or actuators can be considered in two steps: (1) Represent the plant as an FIR filter and (2) Construct an adaptive filter to determine the control. For a discrete signal $u(k)$, a digital filter of order $L+1$ for the plant is

$$
y(k)=\sum_{\ell=0}^{L} h_{\ell}(k) u(k)
$$




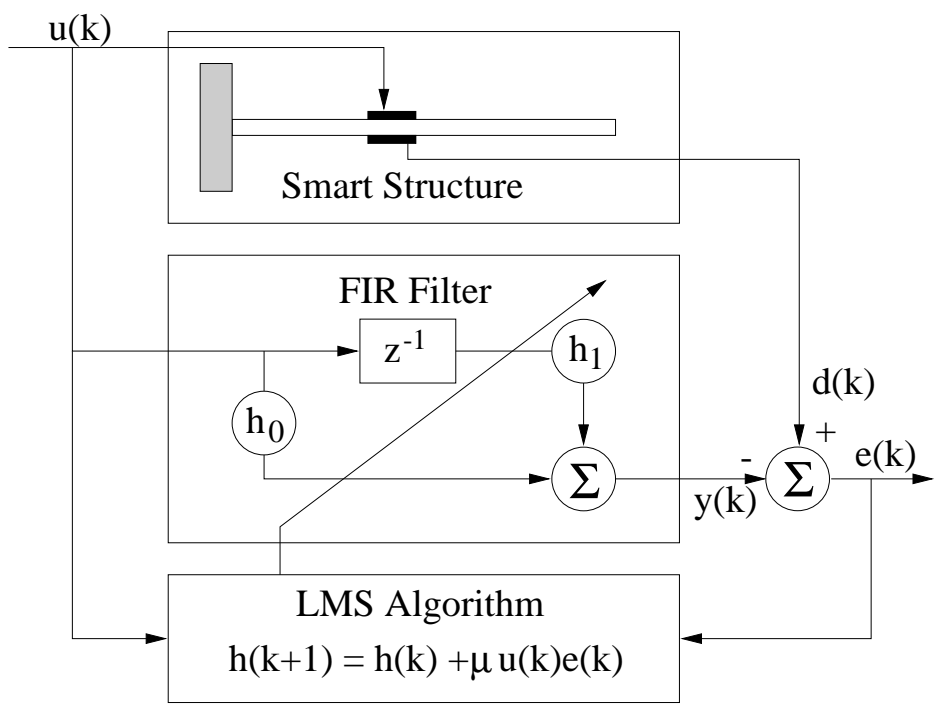

Figure 15: System identification of a smart structure using a two coefficient FIR filter (after [19]).

where $h(k)=\left[h_{0}(k), \cdots, h_{L}(k)\right]$ denotes weights which must be determined through some optimization procedure (e.g., steepest descent, Newton's method, LMS algorithms). As detailed in [19], the minimization of a quadratic performance functional while employing instantaneous estimates of the correlation matrix and cross-correlation vector yields the LMS algorithm

$$
h(k+1)=h(k)+\mu u(k) e(k)
$$

where $e(k)=d(k)-y(k)$ and $\mu$ is chosen so that filter weights approach the optimal Wiener filter (see also [88]). A schematic of the system identification process is depicted in Figure 15. The transversal filter (45) is used both to represent the response of the system to an applied control and to filter reference signals applied to the plant.

The second step is the determination of an adaptive FIR filter for the control. Considering first the case of a single input, the control is represented by the FIR filter

$$
u(k)=\sum_{n=0}^{N} w_{n} r(k-n)
$$

where $r(k-n)$ denotes the reference signal and $w_{n}$ are filter coefficients. From (45), it follows that the output of the plant in response to the control input is

$$
y_{u}(k)=\sum_{\ell=0}^{L} h_{\ell} \sum_{n=0}^{N} w_{n} r(k-n-\ell) .
$$

The total plant output is then represented by the convolution

$$
\begin{aligned}
y(k) & =y_{r}(k)-y_{u}(k) \\
& =y_{r}(k)-\sum_{n=1}^{N} w_{n} r_{u}(k-n)
\end{aligned}
$$




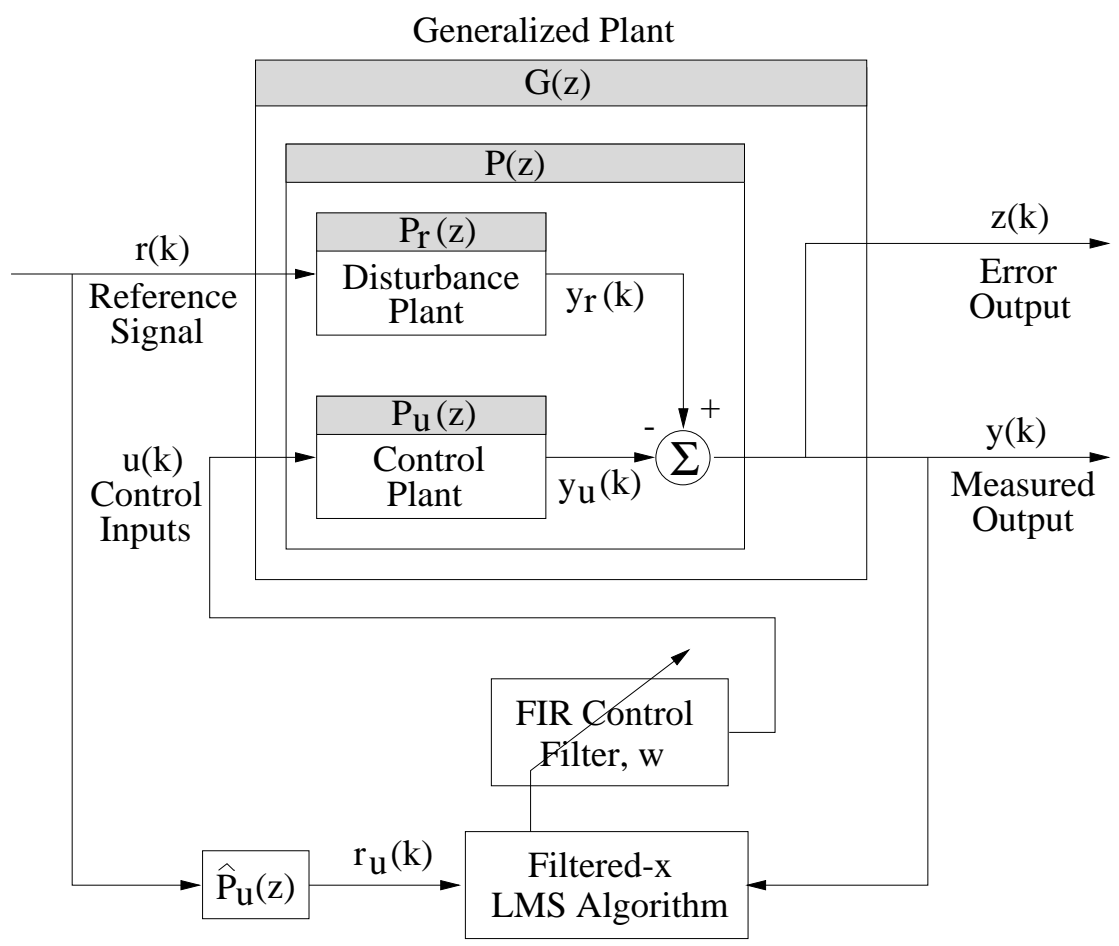

Figure 16: Filtered-x feedforward control algorithm as applied to the smart material structure (after [19]).

where

$$
r_{u}(k-n)=\sum_{\ell=0}^{L} h_{\ell} r(k-n-\ell) .
$$

The signal $r_{u}(k-n)$ is known as the filtered reference signal and from (45), it is observed to be the output of the plant in response to the reference signal $r(k)$. For implementation, this signal can be computed off-line using the LMS algorithm (46) to determine the plant coefficients $h_{\ell}$. This is the filtered-x step in the control computation.

The control coefficients are determined by minimizing the cost functional

$$
J=E\left[y^{2}(k)\right]
$$

using the approximation techniques of Widrow and Stearns [89]. This yields the adaptive filtered-x algorithm

$$
w(k+1)=w(k)+\hat{\mu} r_{u}(k) y(k)
$$

where $\hat{\mu}$ is a second gain parameter which regulates the stability and convergence of the controller. The structure of the algorithm is depicted in Figure 16.

As detailed in [19], the multi-input multi-output case is analyzed similarly and yields the MIMO filtered-x LMS algorithm with $P$ actuators

$$
\mathbf{w}(k+1)=\mathbf{w}(k)+\hat{\mu} \mathbf{r}_{u}(k) \mathbf{y}(k)
$$

where

$$
\mathbf{w}(k)=\left[w_{1}^{T}(k), w_{2}^{T}(k), \cdots, w_{P}^{T}(k)\right]
$$


and

$$
w_{p}(k)=\left[w_{p, 1}(k), w_{p, 2}(k), \cdots, w_{p, N}(k)\right]^{T} \quad, \quad p=1,2, \cdots, P .
$$

Details regarding the construction of the matrix $\mathbf{r}_{u}(k)$ for the filtered reference signal can be found in $[19]$.

\subsubsection{Hybrid Control}

Hybrid control methods can be designed to combine the strengths of feedback and feedforward formulations to improve controller performance in systems subjected to both stochastic and harmonic exogenous forces as well as systems containing modeling uncertainties. Furthermore, the shifting of plant poles through the incorporation of a feedback compensator in the control path can significantly improve convergence times for the feedforward component of the system. Details and software illustrating the implementation of such hybrid methods can be found in [19].

To indicate the performance capabilities of such hybrid approaches, we consider the beam illustrated in Figure 13 with a pair of piezoceramic actuators. A $600 \mathrm{~Hz}$ exogenous force $p$ was applied uniformly along the beam to simulate a periodic driving pressure field. The beam was assumed to have length $\ell=.46 \mathrm{~m}$, width $b=.02 \mathrm{~m}$ and thickness $h=.0016 \mathrm{~m}$, and the patches extended from $x_{1}=.2 \mathrm{~m}$ to $x_{2}=.25 \mathrm{~m}$. The density, stiffness and damping parameters in (22) and (23) were specified to be $\rho(x)=$ $.093+.433 \chi_{p z t}(x) \mathrm{kg} / \mathrm{m}, E I(x)=.491+.793 \chi_{p z t}(x) \mathrm{Nm}^{2}$ and $c_{D} I(x)=.649 \times 10^{-5}+1.255 \times 10^{-5} \chi_{p z t}(x)$ $\mathrm{Nm}^{2}$ sec in accordance with physical parameters identified for similar structures.

A hybrid controller comprised of the feedback law (36) implemented in parallel with the feedforward law (47) was used to determine controlling voltages to the patches given point measurements of the velocity at the point $\bar{x}=3 \ell / 5$. The controlled velocity and adaptation of the weights in the feedforward filter are plotted in Figure 17. It is observed that by 0.1 seconds the weights have converged and by 0.2 seconds, vibrations have been effectively attenuated. When considered in combination with cited references, this illustrates aspects of representative linear control systems which are currently employed for smart structures which utilize piezoceramic actuators in their linear range.

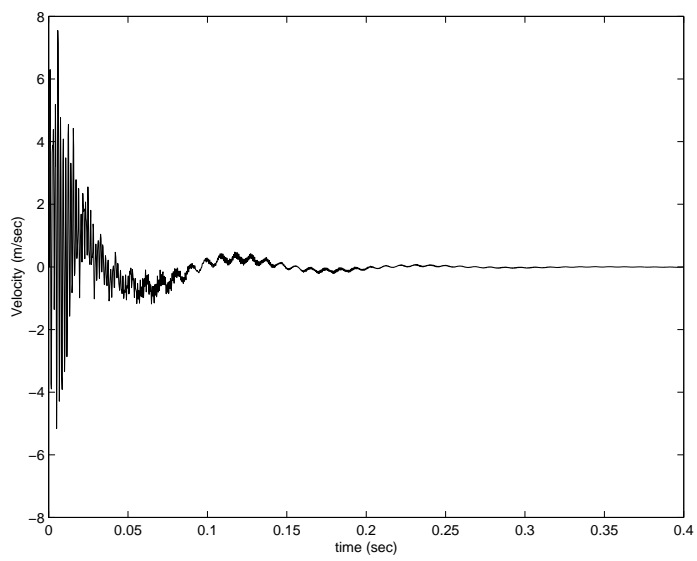

(a)

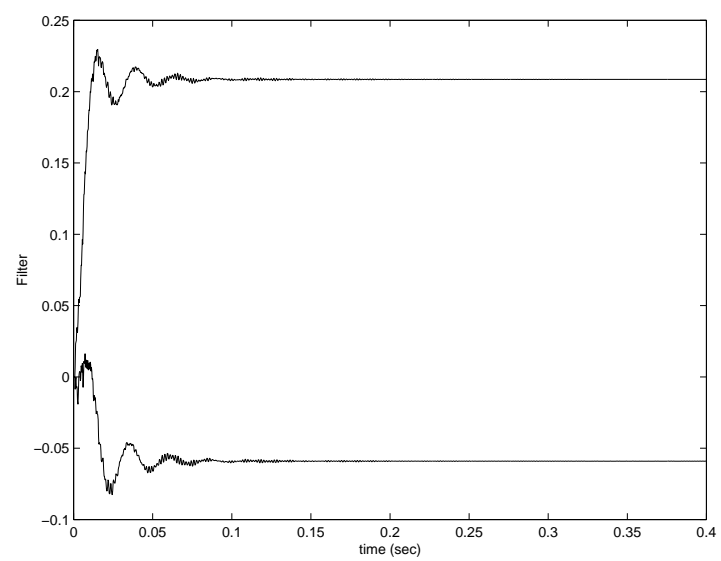

(b)

Figure 17: (a) Beam velocity at the point $\bar{x}=3 \ell / 5$. (b) Adaptation of the filter weights to accommodate the harmonic exogenous force to the beam. 


\section{Magnetostrictive Materials}

Magnetostrictive transducers are increasingly employed in automotive and industrial applications which require the generation of massive forces or strains, or the sensing of magnetic or elastic quantities such as torques or strains. These transducer materials rely on the magnetostrictive effect which is inherent to all ferromagnetic materials but is sufficiently large in compounds such as nickel or Terfenol-D to warrant their use as high performance actuators and sensors. For such compounds, the application of a magnetic field produces strains in the material while conversely, strains produced by external forces generate changes in the magnetic state. Because the magnetostriction is an inherent property of the materials, their performance does not degrade over time as can be the case with piezoelectric compounds.

To motivate modeling and control issues, we first outline certain applications which utilize magnetostrictive actuators and sensors. We then summarize pertinent material characteristics as a prelude to the development of constitutive and hysteresis models and their incorporation in system models. As in the previous section on piezoelectric and electrostrictive transducers, we conclude with prototypical control designs and directions for future research.

\subsection{Magnetostrictive Transducer Applications}

The effects of magnetostriction have been recognized since the 1840's and devices utilizing these properties have been in existence since the 1860's [38]. In the first half of this century, applications utilizing nickel and other magnetostrictive compounds included telephone receivers, torque sensors, and hydrophones [24]. In 1978, A.E. Clark and co-workers developed Terfenol-D ( $\left.\mathrm{Tb}_{x} \mathrm{Dy}_{1-x} \mathrm{Fe}\right)$ which is classified as a "giant" magnetostrictive alloy due to its capability for generating strains in excess of $1000 \mu \mathrm{L} / \mathrm{L}$. Applications which utilize these alloys include active vibration and noise control, micropositioning in high force regimes, medical and industrial ultrasonics, non-contact torque sensors, and tuned vibration absorbers. The reader is referred to $[24,69]$ for a comprehensive discussion of these applications.

The design of magnetostrictive transducers is rapidly evolving as the capabilities of these materials become better understood. A common design for numerous structural applications, which motivates pertinent modeling and control issues, is the piston actuator depicted in Figure 18. Strains in the Terfenol-D rod are produced when fields generated by current through the solenoid cause the rotation of magnetic moments in the manner illustrated in Figure 19. The sensor capabilities of the transducer are produced by the opposite effect in which stresses to the rod realign moments which produces subsequent changes in the magnetization. The prestress mechanism serves two roles: it increases the percentage

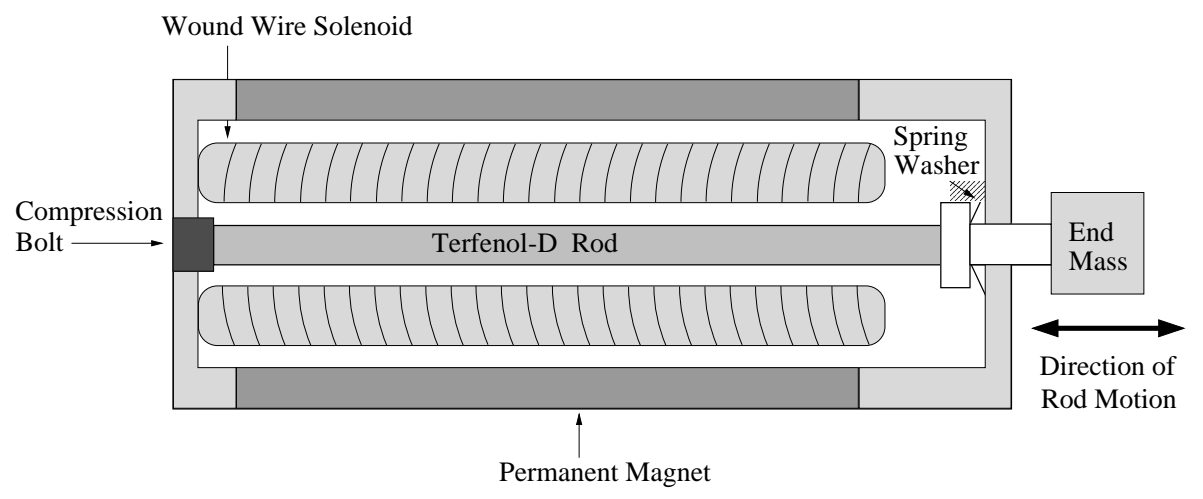

Figure 18: Cross section of a prototypical Terfenol-D magnetostrictive transducer. 


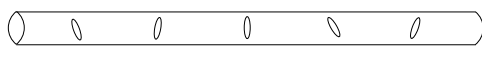

(a)

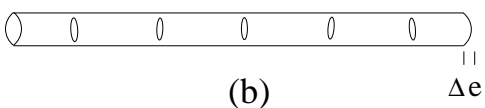

(b)

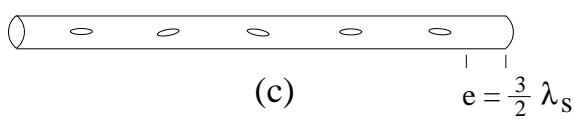

Figure 19: Magnetic domains in the Terfenol-D rod; (a) Orientation of unstressed rod in absence of applied magnetic field. (b) Orientation of pre-stressed rod with no applied field. (c) Orientation of pre-stressed rod when field is applied in direction of longitudinal rod axis.

of moments which are oriented perpendicular to the rod axis and it maintains the rod in a state of compression. The bias fields required to attain bidirectional strains and improved linearity are provided either by a surrounding permanent magnet or through a prescribed DC current to the solenoid. In spite of the fact that the permanent magnet adds bulk, it is typically employed since it reduces ohmic heating to the coil and provides additional flexibility for shaping the flux path to optimize performance.

The manner through which the transducer can be employed in acoustic and structural systems is illustrated in Figure 20. As indicated in (a) and (b), the transducers can be used to both sense vibrations in structures or industrial equipment and provide the force or strain inputs required to attenuate vibrations. A current area of development is the utilization of magnetostrictive materials for non-contact torque sensing. Figure 20(c) illustrates the coupling of the transducer with an ultrasonic horn for applications ranging from the cleaning of intricate or inaccessible machinery to the catalysis of chemical reactions [24].

A second class of magnetostrictive transducers includes flextensional devices analogous to those described in Section 2.1, inchworm devices and rotational motors. The flextensional designs are similar

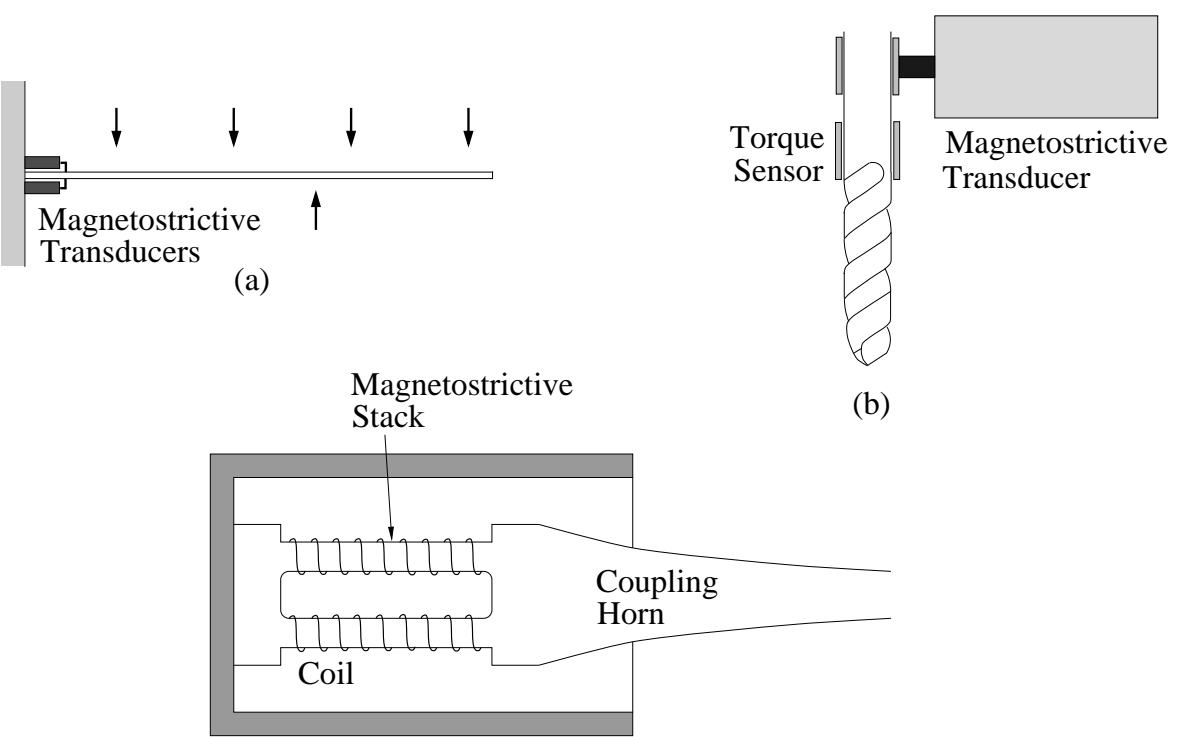

(c)

Figure 20: Applications utilizing magnetostrictive transducers. (a) Vibration sensing and attenuation, (b) Torque sensing and vibration attenuation in a milling machine, (c) Ultrasonic horn (after [24]). 

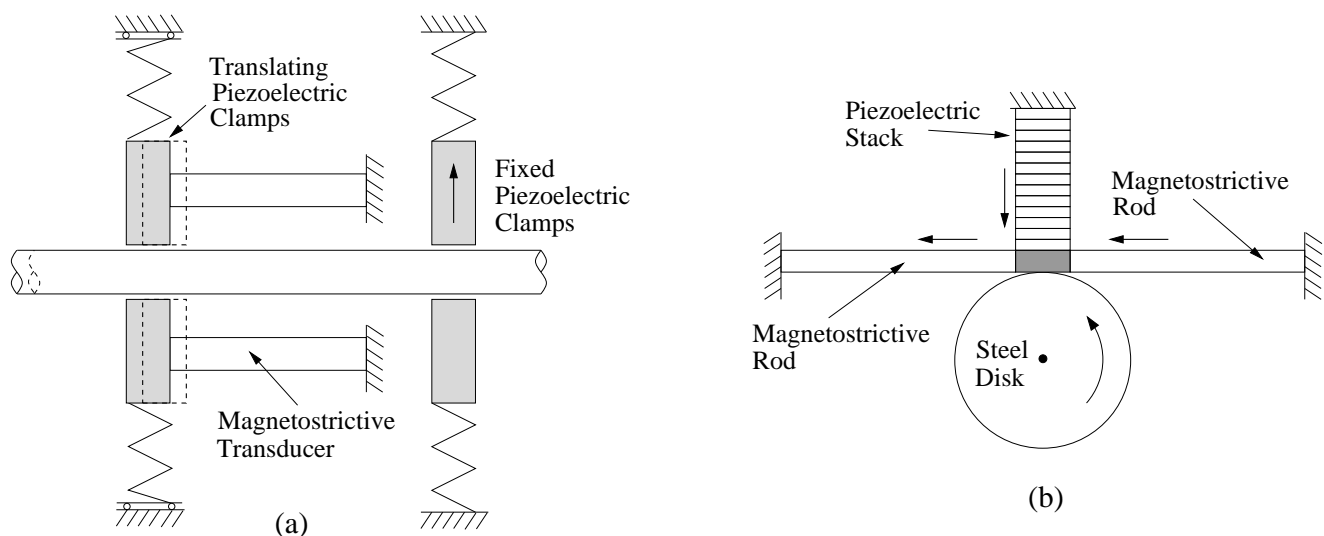

(b)

Figure 21: Hybrid magnetostrictive-piezoceramic transducers. (a) Inchworm actuator (after [59]) and (b) Rotational motor (after [86]).

to those depicted in Figure 4 but utilize a magnetostrictive core rather than PZT or PMN stacks. The magnitude of generated forces in combination with the dual actuating and sensing capabilities of the materials makes the transducers potentially advantageous in hydrostatic applications including sonar transduction and underwater imaging. Hybrid devices employing magnetostrictive drive rods and piezoceramic clamps are depicted in Figure 21. In addition to the forces provided by the magnetostrictive materials, the intrinsic nature of the magnetomechanical coupling provides the possibility for eliminating the variability and aging associated with conventional hydraulic and electromagnetic motors.

A comparison of magnetostrictive transducers with the piezoelectric and electrostrictive devices discussed in Section 2.1 indicates that while they exhibit superior performance in certain applications, they are bulkier than their ferroelectric counterparts due to the required solenoid and housing. Hence they are currently employed in regimes where giant forces or strains are required but weight is not an issue. The range of applications will almost surely increase, however, as materials such as magnetocomposites are perfected.

While there are variations among magnetostrictive transducer designs, certain modeling and control issues are common to all applications. Hysteresis is present to some degree in all magnetostrictive materials and the constitutive relations between the magnetization and strain exhibits quadratic behavior at low drive levels and saturates at high drive levels. Hence biasing fields or stresses are often applied to produce bidirectional strains and improve linearity. In addition to quantifying the nonlinear and hysteretic relations between the field, magnetization and strain, it is necessary to characterize the electromagnetic relations between the current input to the solenoid and the field generated at various points in the rod, the frequency-dependent losses, the coupled magnetomechanical dynamics of the structure, and thermal effects. While aspects of this have been accomplished for specific transducer configurations, comprehensive models which incorporate all of these mechanisms are lacking. In Sections 3.3 and 3.4, we summarize certain constitutive and hysteresis models and illustrate their incorporation in system models for smart structures. The development of commensurate control methods is then outlined in Section 3.5.

\subsection{Material Behavior}

The hysteresis and nonlinear constitutive behavior of magnetostrictive materials are due in part to the domain structure inherent to ferromagnetic compounds. We summarize here material attributes which directly pertain to the performance of the compounds in transducer design both to illustrate intrinsic 
properties of the materials and to motivate the models summarized in Section 3.3. We note that domain mechanisms in ferromagnetic materials are analogous in many ways to the domain attributes which produce hysteresis in ferroelectric materials (Section 2.3) and when possible, we try to consider models and control designs which encompass both sets of compounds.

The description of crystalline attributes of the materials is facilitated by vector conventions in which planes are denoted by round brackets and directional indices are represented by square brackets. Hence the faces of a cube are denoted by $(100),(010),(001),(\overline{1} 00),(0 \overline{1} 0),(00 \overline{1})$ while the vertices are specified by $[100],[010],[001],[\overline{1} 00],[0 \overline{1} 0],[00 \overline{1}]$. In both cases, $\overline{1}$, indicates a negative direction. Finally, pointed brackets are used to summarize an entire set of indices (e.g., $\langle 100\rangle$ represents the vertices of the cube).

\subsubsection{Ferromagnetic Domain Structure and Hysteresis}

For current smart structure applications, the most widely employed magnetostrictive material is TerfenolD. As detailed in [16] and depicted in Figure 22, Terfenol-D crystals are grown in dendrite sheets oriented in the $[11 \overline{2}]$ direction. At room temperature, the easy axes for Terfenol-D lie approximately in the $\langle 111\rangle$ direction and the greatest strains occur when the magnetization $M$ rotates from [111] to [11̄]].

The changes in magnetism which result from an applied field $H$ are primarily due to two mechanisms: the rotation of moments and the movement of domain walls. To illustrate, consider a demagnetized compound which is subjected to a magnetic field oriented in the [11̄] direction as shown in Figure 23. At low field levels, the primary change in magnetization is due to domain wall motion so that favorably

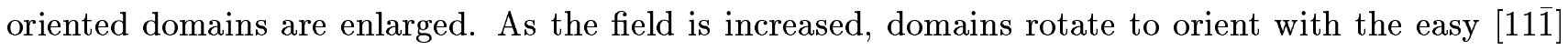
axis. This produces a burst region in the $H-M$ or $H$-e curve in which small changes in field produce large changes in magnetization or strain (see Figure 24). From a design perspective, biasing into this region can be used to optimize performance. In the final stage depicted in Figure 23, the material acts as a single domain as dipoles rotate from the easy axis into the direction of the applied field. This produces the saturation observed in Figure 24.

For low field deviations about a bias field level, the relation between the field and magnetization is approximately linear as illustrated in Figure 24a. At higher field levels, however, magnetostrictive materials typically exhibit hysteresis of the form depicted in Figure 24b. As with ferroelectric compounds

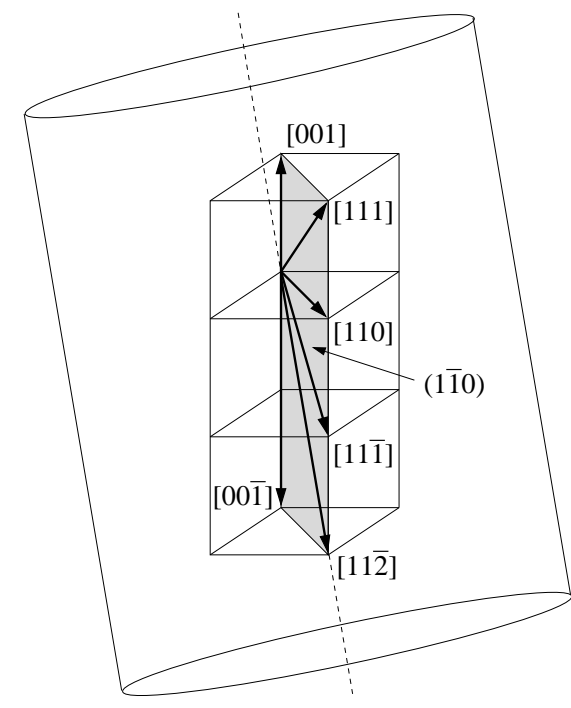

Figure 22: Orientation of Terfenol-D crystals. 


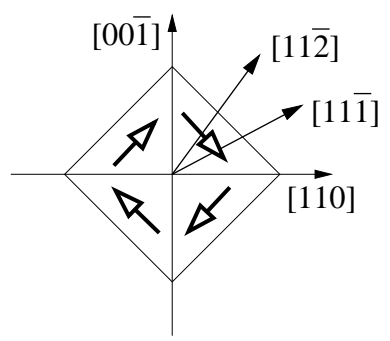

(a)

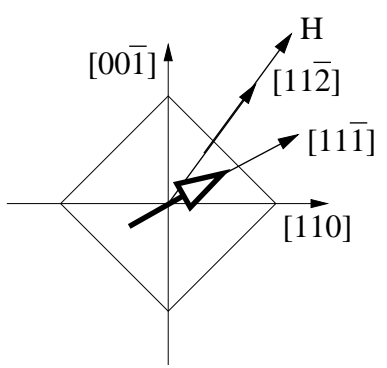

(c)

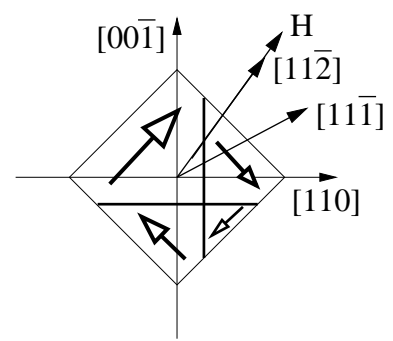

(b)

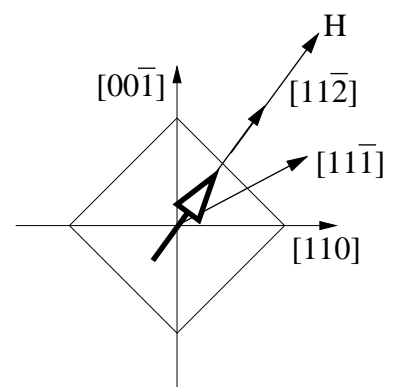

(d)

Figure 23: Magnetization process due to an applied field $H$. (a) Demagnetized state and (b) growth of domains due to domain wall motion. (c) Rotation of domains to the easy axis and (d) Rotation of domains to align with the applied field.

(see Section 2.2), this hysteresis can be due to the irreversible impediment of domain wall movement by inclusions or pinning sites in the material. Details regarding the sources of hysteresis in ferromagnetic materials can be found in $[10,22,44]$.

Strains, or magnetostriction, are generated by the material when dipoles rotate to align with an applied field. For general configurations, the magnetomechanical coupling which produces these strains is highly complex and dependent upon factors such as the applied stress and crystalline anisotropies. However, in the case of materials in which a prestress perpendicular to the dipole direction is sufficiently

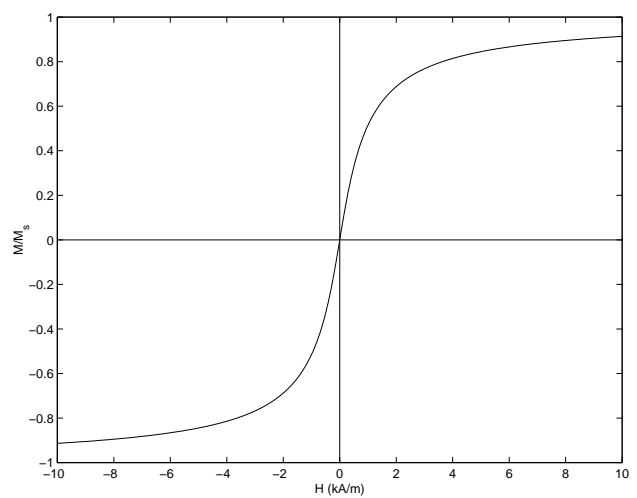

(a)

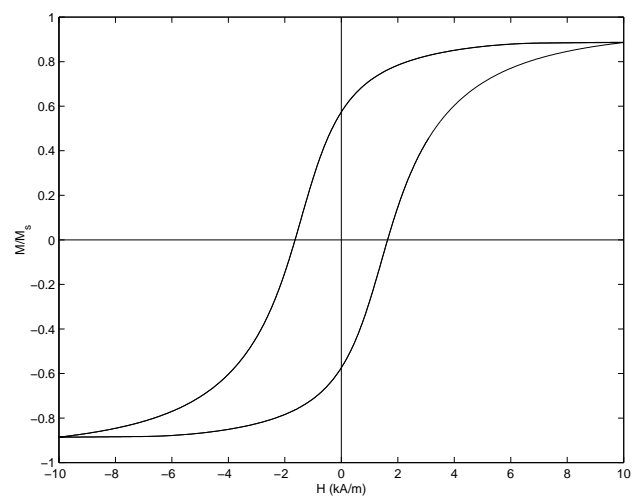

(b)

Figure 24: (a) Anhysteretic magnetization curve. (b) Ferromagnetic hysteresis present at high drive levels. 
large to dominate crystalline anisotropies, the strains are due primarily to domain rotation in the manner depicted in Figure 19. For this regime, the magnetostriction $\lambda$ can be modeled by the quadratic relation

$$
\lambda=\frac{3}{2} \lambda_{s}\left(\frac{M}{M_{s}}\right)^{2}
$$

where $\lambda_{s}$ and $M_{s}$ respectively denote the saturation magnetostriction and magnetization. As discussed in $[13,44]$, the prestress levels which optimize transducer performance are often of a magnitude so that stress anisotropies dominate crystalline anisotropies and the relation (48) adequately models the free strains generated by the material.

\subsection{Transducer Models}

A large number of modeling techniques have been employed to characterize the behavior of magnetostrictive materials in various operating regimes. These include linear and higher-order anhysteretic models as well as micromagnetic, Preisach, domain rotation and domain wall models for the hysteresis present at higher drive levels. We outline here models which have been employed for control design and direct the reader to various references for more comprehensive perspectives concerning individual modeling techniques.

\subsubsection{Anhysteretic Models}

For low drive level applications in which hysteresis is minimal, anhysteretic (hysteresis-free) models analogous to those employed for piezoceramic and electrostrictive compounds have been adopted for magnetostrictive materials. In most control applications, a bias field produced either by a surrounding permanent magnet (see Figure 18) or DC field to the solenoid is used to attain bidirectional strains and improve linearity. For low field levels, the linear model

$$
\begin{gathered}
B_{i}=d_{i j k} \sigma_{j k}+\mu_{i j}^{\sigma} H_{j} \\
e_{i j}=s_{i j k \ell}^{H} \sigma_{k \ell}+d_{k i j} H_{k}
\end{gathered}
$$

is often employed. A comparison with (1) reveals the similarity with the linear piezoelectric model. The stress, strain and compliance tensors $\sigma, e$ and $s^{H}$ are identical to those employed in Section 2.3 while the tensor $d$ is analogous to the piezoelectric strain tensor. Finally, $\mu^{\sigma}$ denotes the permeability at constant stress. The model (49) has the advantage of simplicity and linearity but does not incorporate the nonlinear constitutive behavior or hysteresis present in many high drive level smart structure applications.

Saturation phenomena, temperature effects, and the quadratic relation between the magnetization and free strains summarized in (48) are incorporated in the higher-order anhysteretic model

$$
\begin{aligned}
& H_{k}=-2 Q_{i j k \ell}^{T} M_{\ell} \sigma_{i j}+\frac{M_{k}}{k_{1}|M|} \operatorname{arctanh}\left(|M| / M_{s}\right)+P_{k}^{\sigma} \Delta T \\
& e_{i j}=s_{i j k \ell}^{M, T} \sigma_{k \ell}+Q_{i j k \ell}^{T} M_{k} M_{\ell}+\alpha_{i j}^{M} \Delta T
\end{aligned}
$$

of Duenas, Hsu and Carman [28]. Here $Q^{T}$ is a fourth rank tensor of magnetostrictive coefficients, $T$ is the temperature, $\alpha^{M}$ is a coefficient of thermal expansion, $P^{\sigma}$ is a pyromagnetic coefficient and $k_{1}$ is a constant. In each case, the superscript denotes quantities which are fixed. A comparison with (4) for $\mathcal{F}$ given by (9) illustrates that (50) is analogous to the quadratic electrostriction model in which the Ising spin relation (7) is used to characterize the anhysteretic polarization. 
The higher order model quantifies certain aspects of the nonlinear constitutive behavior but can incorporate hysteresis only through the use of complex model parameters. Techniques for modeling the hysteresis in the magnetization $M$ in the first (converse) relation in (50) are addressed next.

\subsubsection{Hysteresis Models}

Hysteresis models for magnetostrictive materials range from micromechanical models to phenomenological characterizations. Micromechanical models quantify the coupled changes in magnetization and strain through first principles formulations of the elastic and magnetostatic states [11, 42]. While this can produce highly accurate material models, the large number of required parameters currently precludes the real-time implementation of controllers which employ these models. Preisach models for magnetic and magnetostrictive materials have been developed by numerous researchers $[1,70,76]$, and the advantages and disadvantages of this approach are similar to those discussed for ferroelectric Preisach models (see Section 2.3). Domain and domain wall models include classical and modified anisotropy domain rotation models $[46,84]$ and the domain wall theory of Jiles and Atherton [45] which motivated the ferroelectric hysteresis model discussed in Section 2.3 for piezoceramic and electrostrictive models.

As detailed in Section 2.3, the domain wall theory models hysteresis by quantifying the energy required to bend and translate domain walls pinned at inclusions inherent to the material. This yields irreversible and reversible components whose sum is the total polarization in ferroelectric materials or magnetization in ferromagnetic materials.

From the theory of Jiles and Atherton [45], the irreversible magnetization

$$
M_{i r r}=M_{a n}-k \frac{d M_{i r r}}{d H_{e}}
$$

is specified by quantifying losses from the minimum energy anhysteretic magnetization $M_{a n}$. The effective field is given by $H_{e}=H+\alpha M$ and the anhysteretic magnetization is modeled by the Langevin equation

$$
M_{a n}=M_{s}\left[\operatorname{coth}\left(\frac{H_{e}}{a}\right)-\left(\frac{a}{H_{e}}\right)\right]
$$

where $\alpha$ and $a$ are parameters specifying the slope of the anhysteretic curve. The parameter $k$ quantifies the average energy required to change moment orientations and hence break pinning sites. The formulation of the irreversible magnetization in terms of the applied field yields

$$
\frac{d M_{i r r}}{d H}=\widetilde{\delta} \frac{M_{a n}-M_{i r r}}{k \delta-\alpha\left(M_{a n}-M_{i r r}\right)}
$$

where $\delta=\operatorname{sign}(H)$ and $\widetilde{\delta}$ is defined in a manner analogous to (12).

The Jiles-Atherton theory characterizes the magnetization due to the bending of domain walls through the algebraic relation

$$
M_{r e v}=c\left(M_{a n}-M_{i r r}\right)
$$

where $c$ quantifies reversible domain wall effects. The total magnetization is then specified by

$$
M=M_{i r r}+M_{r e v}
$$

We note that the formulation of this model is identical to the ferroelectric hysteresis model (14). This permits both a unified theory for characterizing hysteresis in the two classes of materials and the development of control algorithms which accommodate both classes of transducers. 
An alternative formulation of the model which facilitates inversion can be obtained by consolidating the irreversible and reversible magnetizations to obtain the differential equation

$$
\begin{aligned}
& \frac{d M}{d H}=\mathcal{F}(H, M) \\
& M\left(H_{0}\right)=M_{0}
\end{aligned}
$$

where

$$
\mathcal{F}(H, M)=\frac{1}{1+c M_{s} \alpha \frac{\partial \mathcal{L}}{\partial H}\left(\frac{H+\alpha M}{a}\right)}\left\{\frac{\widetilde{\delta}\left[M_{s} \mathcal{L}\left(\frac{H+\alpha M}{a}\right)-M\right]}{k \delta-\tilde{\alpha}\left[M_{s} \mathcal{L}\left(\frac{H+\alpha M}{a}\right)-M\right]}-c M_{s} \frac{\partial \mathcal{L}}{\partial H}\left(\frac{H+\alpha M}{a}\right)\right\} .
$$

The Langevin function is defined by

$$
\mathcal{L}(z) \equiv \operatorname{coth}(z)-\frac{1}{z}
$$

The time-dependent dynamics of the magnetization can then be specified through the chain rule

$$
\frac{d M}{d t}=\mathcal{F}(H, M) \frac{d H}{d t}
$$

We note that in the formulation outlined here, frequency-dependent eddy current losses are ignored so the relation (53) should be employed in regimes where these effects are minimal.

The magnetization model (51) or (52) can be combined with the constitutive relation (50) to quantify the strains generated by the transducer when hysteresis is significant. Experimental validation of this model for a prototypical transducer of the type depicted in Figure 18 can be found in [25, 26].

The hysteresis model in the form (52) is also amenable to inversion through consideration of the complementary differential equation

$$
\begin{aligned}
& \frac{d M^{-1}}{d H}=\frac{1}{\mathcal{F}\left(M^{-1}, H\right)} \\
& M^{-1}\left(H_{0}\right)=M_{0}^{-1}
\end{aligned}
$$

The experimental validation of the inversion procedure is detailed in [83] and numerical examples describing its role in control design will be outlined in Section 3.5.

\subsection{System Models}

To illustrate the effect of the nonlinear input on a smart structure which employs magnetostrictive transducers, we consider a prototypical beam with end-mounted transducers as depicted in Figure 20a. The description of the beam was provided in Section 2.4 and we focus here solely on the Terfenol-D transducers which are driven diametrically out-of-phase to produce pure bending moments in the beam. As detailed in [14], this setup has been employed in experiments to ascertain capabilities and properties of the transducers.

For modeling purposes, the cross-sectional area and Young's modulus for the Terfenol-D rod are denoted by $A_{m a g}$ and $E^{H}$ while the length of the connecting rod is $\ell_{r}$. As detailed in [75], the transverse displacements of the beam are modeled by (21) with the density $\rho$, stiffness $E I$ and damping coefficient $c_{D} I$ modified to incorporate material properties of the Terfenol rod and connecting bar. For a system in which the magnetization is biased to obtain bidirectional strains, the quadratic relation (48) yields the free strains

$$
e(t)=\frac{3 \lambda_{s}}{2 M_{s}^{2}}\left[M^{2}(t)+2 M(t) M_{s}\right]
$$


The external moment generated by the transducers is then

$$
\mathcal{M}_{\text {ext }}(t, x)=\mathcal{K}^{M}\left[M^{2}(t)+2 M(t) M_{s}\right] \chi_{\text {rod }}(x)
$$

where $\mathcal{K}^{M}=\left(3 \lambda_{s} / M_{s}^{2}\right) A_{m a g} E^{H}\left(h / 2+\ell_{r}\right)^{2}$ and the magnetization $M$ is modeled by the hysteresis relation $(51)$ or $(52)$.

The formulation of the model (21) in weak form and approximation using the Galerkin expansion (28) yields the first order system

$$
\begin{aligned}
& \dot{y}(t)=A y(t)+[B(u)](t)+g(t) \\
& y(0)=y_{0}
\end{aligned}
$$

where the nonlinear input term has the form

$$
[B(u)](t)=\left[M^{2}(H(t))+2 M(H(t)) M_{s}\right] b .
$$

The system matrices and vectors $y, A, r$ and $b$ are defined in (30).

\subsection{Control Formulation}

There are essentially three options for designing control methods for systems which employ magnetostrictive transducers. The first is to employ the linear constitutive relations (49) which yields a linear system. This provides a setting in which feedforward and feedback methods of the type illustrated in Section 2.5 can be employed, but is accurate only at low drive levels. The second option is to determine an inverse model for the transducer which either exactly or approximately compensates for the actuator hysteresis. This strategy will be illustrated here for magnetostrictive transducers and in the context of Preisach models for shape memory alloys in Section 4. The final option is to design a nonlinear control law for the system. This is done in [77] for the structural system described here and the examples in that investigation provide a baseline for determining the effectiveness of the feedback controls which incorporate an inverse compensator.

\subsubsection{Control Example}

We consider two techniques for formulating a linear control problem for the structure and transducer setup described in Section 3.4. In the first case, we simply linearize the operator $B(u)$ about a given field level $u=H$. This technique is commonly employed when magnetostrictive transducers are employed at low drive levels with magnetic biases and is applicable only when the transducer is operated in the nearly linear range. The second technique is based upon the filtering of control inputs using the inverse compensator. This control method is applicable throughout the operational range of the transducer. Details regarding this example are provided in [78].

The spatially uniform exogenous force

$$
g(t, x)=g_{0}[\sin (14 \pi t)+\sin (26 \pi t)]
$$

was applied throughout the time interval $[0,2.5]$ to simulate a periodic pressure field on the beam. The uncontrolled trajectories at the point $\bar{x}=3 \ell / 5$ for $g_{0}=1$ are plotted in Figures 25b and 26a while the trajectories obtained with $g_{0}=100$ are plotted in Figures 25d and 26b Both cases exhibit a beat phenomenon due to the close proximity of the $7 \mathrm{~Hz}$ driving frequency with the $6.1 \mathrm{~Hz}$ natural frequency of the beam. 


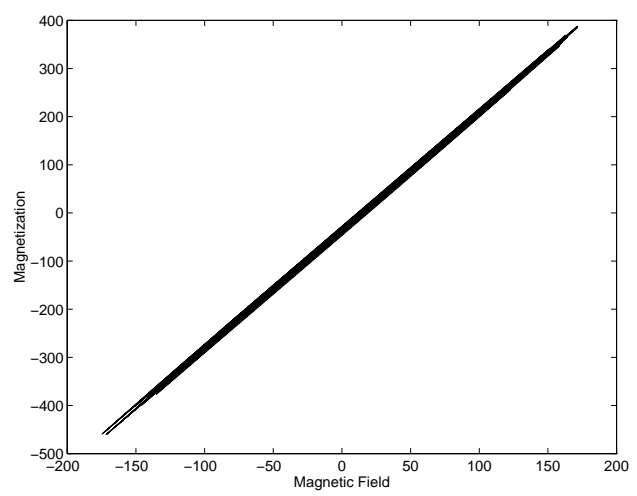

(a)

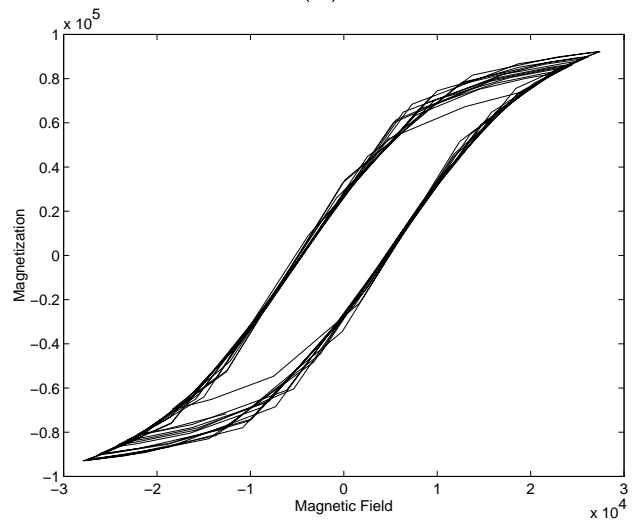

(c)

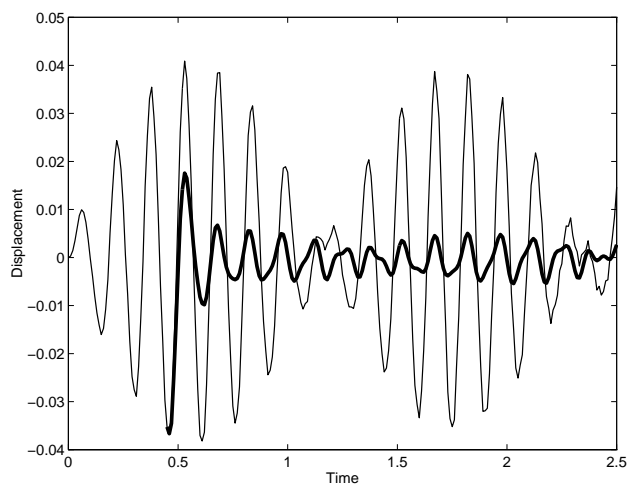

(b)

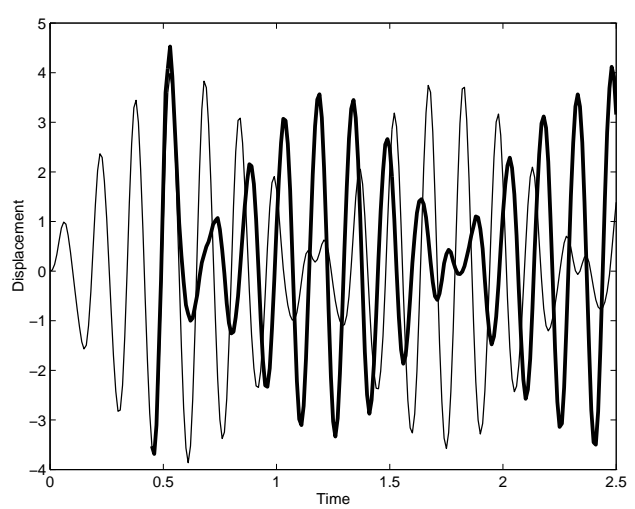

(d)

Figure 25: Feedback of the linear law (42) into the nonlinear system (56). Relationship between magnetic field $H$ and magnetization $M$ for (a) $g_{0}=1$ and (c) $g_{0}=100$. Uncontrolled ( $\longrightarrow$ ) and controlled ( $\left.\longrightarrow\right)$ beam trajectories at $\bar{x}=3 \ell / 5$ for (b) $g_{0}=1$ and (d) $g_{0}=100$.

An open loop control input was computed using (42) with full state measurements $x_{c}=x$, and applied to the hysteretic system (56) at time $t=0.45$. The resulting controlled beam trajectories at the $\bar{x}$ are plotted in Figures $25 \mathrm{~b}$ and $25 \mathrm{~d}$ and the relationship between the input magnetic field $H=u$ and the resulting magnetization $M$ for the two cases are given in Figures 25a and c. The results for $g_{0}=1$ illustrates that the method is very effective at low drive levels where the linear model is accurate. At the high drive levels at which the actuators are advantageous, however, the input $B(u)$ introduces significant hysteresis which acts as a time delay to the system. The result is a loss in control authority which is sufficiently severe to produce controlled beam trajectories which are larger in magnitude at certain times than the uncontrolled trajectories.

To obtain a control based on the inverse compensator, we then employed the input operator $B=b$ where $b$ is defined in (30). This yields the prescribed input $u_{d}$. To obtain the optimal control for the original system (56), the signal $u_{d}$ is then filtered through the inverse

$$
v(t)=M^{-1}\left(M_{s}+\sqrt{M_{s}^{2}+u_{d}(t)}\right)
$$

before input to the transducer. An overview of this inversion process is illustrated in Figure 12 in the context of a Preisach model. We note that while this requires the on-line integration of (54) to obtain $M^{-1}$, the operation is significantly less expensive than the online integration of (44) to obtain $z(t)$ or the integration of (43) required to obtain state estimates $x_{c}(t)$ when full state information is unavailable. 


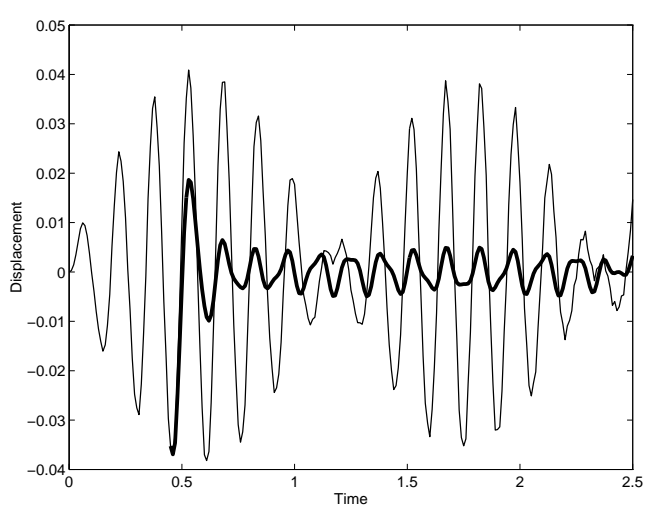

(a)

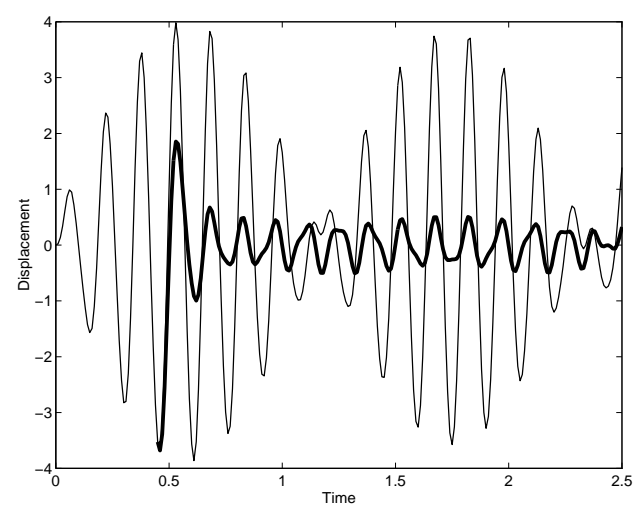

(b)

Figure 26: Uncontrolled beam trajectory (—) at the point $\bar{x}=3 \ell / 5$ and controlled trajectory ( - ) obtained with the filtered input (57); (a) $g_{0}=1$ and (b) $g_{0}=100$.

The controlled trajectories obtained in this manner for $g_{0}=1$ and $g_{0}=100$ are respectively plotted in Figure 26. In this case, there is no loss of control authority at high drive levels, thus illustrating that this approach provides a significant advantage over the control design based on the linearized system. Finally, a comparison with the examples in [77] illustrates that the performance of the controller based on inverse compensation is comparable to that obtained when the functional (40) is minimized subject to the original system (56) but at a fraction of the computational cost.

\section{Shape Memory Alloys}

Shape memory alloys (SMA) comprise a class of metallic compounds which convert thermal energy into mechanical energy. While the underlying mechanisms are complex, the generation of strains in response to changes in temperature or stress can be described as due to crystalline transformations from a high temperature austenite phase to a low temperature martensite phase and vice-versa. For the most commonly employed material Nitinol (Ni: nickel, ti: titanium, nol: Naval Ordinance Laboratory), this can lead to the generation of recoverable strains on the order of $6 \%$. Because the crystalline transformations are driven by changes in temperature, the original shape memory alloys had a low bandwidth which limited their utility for high frequency control applications. Their potential for shape modification or control is significant, however, due to the magnitude of the generated strains. Furthermore, the development of hybrid shape memory alloys has produced transducer materials with improved bandwidth (e.g., see [43]), and applications utilizing these compounds should continue to evolve.

The nature of the martensite to austenite phase transition and the associated change in shape of the SMA produces shape memory effects (SME) which provide the materials with their capabilities as distributed actuators. The phenomenological behavior of the phase transition is illustrated in Figure 27a. The cooling of the SMA from the high temperature austenite phase to the low temperature martensite phase is characterized by a phase transition which starts at a temperature $M_{s}$ and is finished at $M_{f}$. The opposite phase transition occurs as the material is heated through the temperatures $A_{s}$ and $A_{f}$.

To utilize the associated shape memory effects, the SMA are stress-loaded at temperatures $T<A_{s}$ in the martensite phase. This produces the residual strain $e_{r}$ depicted in Figure 27b. These residual strains are recovered by heating the compound past the austenite finish temperature $A_{f}$. For details concerning this process, readers are referred to $[9,31,56]$. 


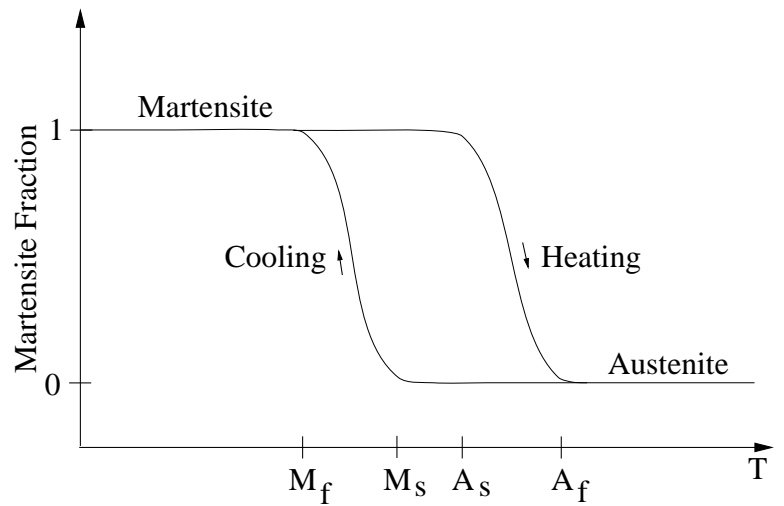

(a)

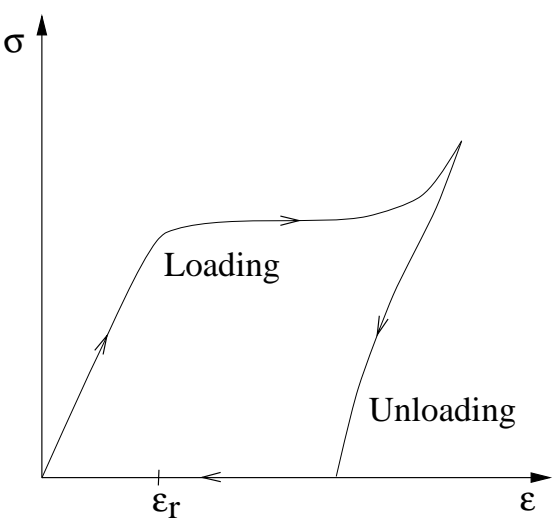

(b)

Figure 27: (a) Martensite-austenite phase transitions in SMA. (b) The residual strain $e_{r}$ for $T<A_{s}$ (after [9]).

The shape memory effects have been utilized in devices such as pipe couplers in which SMA sleeves are heated to seal joints and gas cutoff valves which close in response to elevated temperatures. SMA wires have also been employed as actuators in applications ranging from remote antenna deployment to modifying the camber of a helicopter blade. Their use in a prototypical beam and a reconfigurable wing are depicted in Figure 28. Shape memory alloys currently play an important role as actuators in smart structures requiring the generation of giant strains and their contributions should continue to increase as advances in material science lead to improved bandwidths.

\subsection{Material and System Models}

The modeling of the constitutive behavior of shape memory alloys is difficult due to the large strains and nonlinear phase mechanisms inherent to the materials. The magnitude of the strains precludes the use of infinitesimal elasticity theory and one must consider nonlinear deformation theory based on finite stresses and strains. As illustrated in Figure 27, the constitutive relations are also highly nonlinear and exhibit significant hysteresis due to the nature of the phase transitions. While aspects of this behavior have been quantified through thermodynamic constitutive models $[53,56]$, the state of energy-based models quantifying the dynamics of shape memory alloys lags behind that of piezoelectric, electrostrictive and magnetostrictive materials.

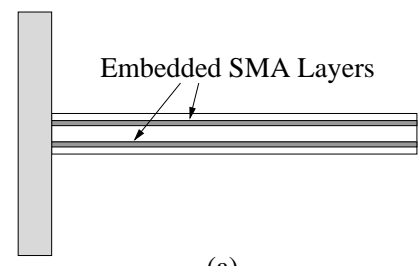

(a)

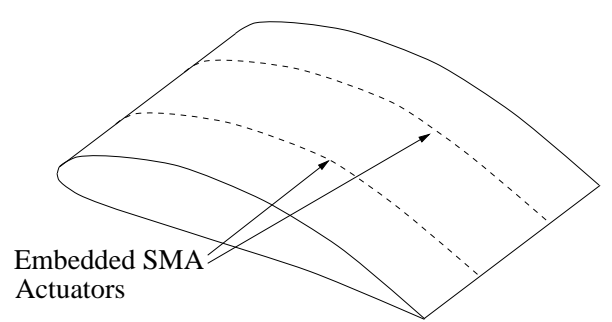

(b)

Figure 28: (a) Prototypical beam with embedded SMA layers. (b) Embedded SMA actuators in a reconfigurable wing (after [87]). 
The complexity of the constitutive behavior and difficulties associated with inverting energy-based models to facilitate linear control design has prompted the development of several phenomenological models for smart structures which utilize shape memory alloys. To illustrate, we summarize the development of a Preisach model for shape memory alloys and outline the manner through which it can be employed in control design.

The construction of a Preisach model quantifying the stresses generated by an SMA wire in response to a change in temperature parallels exactly the Preisach formulation detailed in Section 2.3 for hysteresis in piezoceramic patches, thus attesting to the generality of the method. In this case, the relation between input temperatures and generated stresses is quantified by the Preisach operator $P_{\mu}$ specified in (15) where the input $v=T$ is the temperature and $\xi(s)$ denotes the initial state of the system. The Krasnolselskii-Pokrovskii (KP) kernel (16) is employed to guarantee continuity with respect to time and parameters. As detailed in Section 2.3, the approximation of the measure $\mu$ by a linear combination of Dirac measures and restriction of the Preisach plane to a bounded region $\Delta S$ yields the approximating operators $P_{m}$ specified by (18). The operators $P_{m}$ are amenable to numerical implementation and inversion to facilitate linear control design. Further details regarding the formulation of Preisach models for shape memory alloys are provided in $[6,7,51]$.

To illustrate the use of the Preisach model for characterizing the shape changes produced in a structure by SMA actuators, we consider the beam depicted in Figure 28 which has a pair of embedded SMA layers with cross sectional area $A_{S M A}$. We note that this configuration is treated as a prototypical example in [51] and the following model is from that source. For the coordinate system specified in Figure 13, the dynamics of the beam are modeled by the coupled system

$$
\begin{aligned}
& \rho \frac{\partial^{2} w}{\partial t^{2}}(t, x)+\frac{\partial^{2} \mathcal{M}_{i n t}}{\partial x^{2}}(t, x)=\frac{\partial^{2} \mathcal{M}_{\text {ext }}}{\partial x^{2}}(t, x, T) \\
& \frac{\partial T}{\partial t}(t, x, z)-k\left(\frac{\partial^{2}}{\partial y^{2}}+\frac{\partial^{2}}{\partial y^{2}}\right) T(t, y, z)=k v^{2}(t)
\end{aligned}
$$

with appropriate boundary and initial conditions. Here $w$ and $T$ respectively denote the transverse beam displacement and temperature. As noted in (22), the internal moment is given by $\mathcal{M}_{\text {int }}=$ $E I \frac{\partial^{2} w}{\partial x^{2}}+c_{D} I \frac{\partial^{3} w}{\partial x^{2} d t}$. The density $\rho$, stiffness $E I$, and Kelvin-Voigt damping $c_{D} I$ coefficients are treated as effective parameters which incorporate the material properties of the beam and SMA layer. In the heat equation, $k$ denotes the thermal conductivity and $v(t)$ is the current applied to the shape memory alloy. The external moments generated by the shape memory layer are modeled by the Preisach characterization

$$
\mathcal{M}_{e x t}(t, x, T)=r(x) \int_{\bar{S}}\left[k_{s}(M(T), \xi(s)](t) d \mu(s)\right.
$$

where $r(x)$ is a shape function specifying the spatial stress distribution and

$$
[M(T)](t)=\frac{1}{A_{S M A}} \int_{A_{S M A}} T(t, y, z) d y d z
$$

quantifies the effective temperature in the layer. Further details concerning this model and the identification of the measure $\mu(s)$ are provided in [51].

For control implementation, the infinite dimensional models (58) and (59) must be approximated. This can be accomplished by formulating the system in weak form and employing Galerkin representations analogous to those illustrated in Sections 2.4 and 3.4. The final matrix system will be in the 
form

$$
\begin{aligned}
& \dot{y}(t)=A y(t)+[B(u)](t)+g(t) \\
& y(0)=y_{0}
\end{aligned}
$$

with the structure of the nonlinear input term dictated by the Preisach model (59).

\subsection{Adaptive Control}

The characterization of hysteresis inherent to shape memory alloys using the Preisach model (18) requires the determination of the weights $\theta=\left[\alpha_{n_{1}}, \cdots, \alpha_{n_{m}}\right]$ through either on-line or off-line means. Off-line identification can reduce the computational overhead for control implementation but does not provide a mechanism for tuning parameters to accommodate changing system conditions. This capability can be provided by adaptive identification and control methods. The utilization of adaptive controllers which update parameters in accordance with system outputs is also compatible with the goal in certain smart structure applications of modifying structural properties to accommodate dynamic exogenous forces or changing environmental conditions. We outline here basic mechanisms for constructing a model reference adaptive control (MRAC) in the context of shape memory alloys. The reader is referred to [40] for details concerning the underlying theory and [85] for a complete discussion concerning the implementation of these methods in a variety of systems having actuator and sensor nonlinearities.

The basic configuration of a model reference adaptive controller is illustrated in Figure 29. For fixed inverse compensation, the inverse $B^{-1}$ or approximate inverse $\widehat{B}^{-1}$ is determined off-line and used to cancel the hysteresis and nonlinearities associated with $B$. For the approximate inverse $\widehat{B}^{-1}$, the signal $u$ to the plant is approximately the desired signal $u_{d}$ specified by the control law.

As detailed in $[40,85]$, the control $u_{d}$ is formulated as

$$
u_{d}(t)=\theta_{1}^{* T} \omega_{1}(t)+\theta_{2}^{* T} \omega_{2}(t)+\theta_{3}^{*} y(t)+\theta_{4}^{*} r(t)
$$

where $\theta_{1}^{*}, \theta_{2}^{*}$ are vector-valued and $\theta_{3}^{*}, \theta_{4}^{*}$ are real-valued parameters. The control vectors $\omega_{1}(t)$ and $\omega_{2}(t)$ are specified by

$$
\omega_{1}(t)=K_{1}(D) u_{d}(t), \omega_{2}(t)=K_{2}(D) y(t)
$$

where $K_{1}(D)$ and $K_{2}(D)$ are filters compatible with the form of the nonlinearity (e.g., see [85, page 57]). Following the convention employed in [85], $D$ represents the time differentiation operator or Laplace

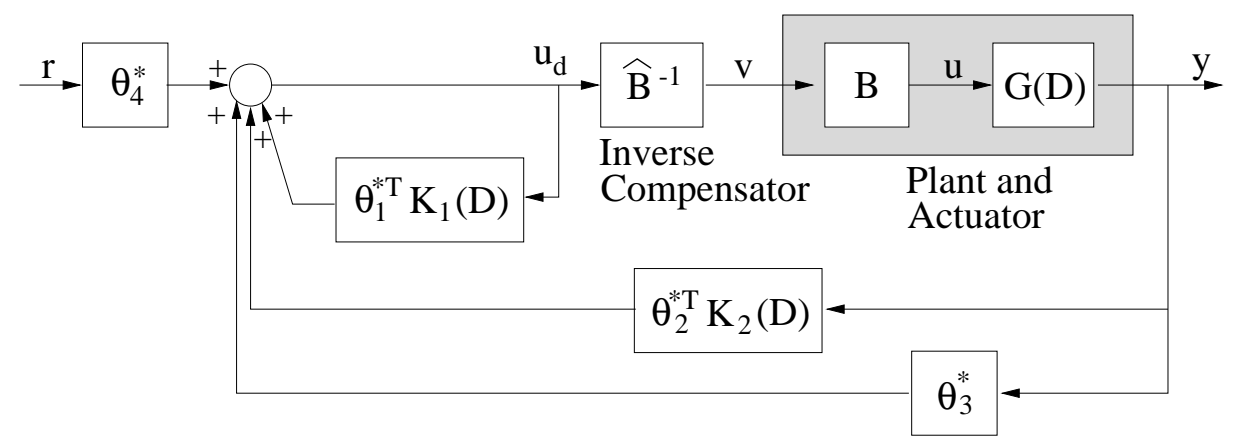

Figure 29: Model reference adaptive control design for a linear plant with a nonlinear actuator and inverse compensator (after [85]). 
transform variable in continuous time or the time advance operator or z-transform in discrete time. The gains $\theta_{1}^{*}, \theta_{2}^{*}, \theta_{3}^{*}, \theta_{4}^{*}$ are specified in a manner which guarantees that closed loop signals are bounded and tracking errors converge exponentially to zero.

While fixed inverse compensation is effective in certain regimes, it does not provide the capability for tuning the approximate inverse $\widehat{B}^{-1}$ to accommodate changing operating conditions or to optimize performance. This motivates the consideration of adaptive control laws which systematically update the parameters $\theta=\left[\alpha_{n_{1}}, \cdots, \alpha_{n_{m}}\right]$ specifying the inverse. As detailed in [40,85], this can be accomplished through a variety of methods including gradient and Lyapunov-type updating laws.

The generality of this approach makes it feasible for several hysteresis models and their inverses. It is illustrated for a general Preisach model in [85] and numerical examples illustrating model reference control of SMA modeled using fixed inverses based on the KP kernel are provided in [51]. Finally, the adaptive updating of parameters in the $\mathrm{KP}$ kernel is experimentally validated for both periodic and random stress variations in [87]. This latter reference also illustrates that PI controllers which ignore hysteresis are effective at very low frequencies $(<2 \mathrm{~Hz})$ but produce large overshoots and inaccurate tracking at higher frequencies. This reiterates the necessity of accommodating the hysteresis in the controller to fully utilize shape memory alloys in smart structure design.

\section{Concluding Remarks}

The emphasis in this review on piezoelectric, electrostrictive, magnetostrictive and shape memory alloys reflects their prominence in current smart structures. This list is by no means complete, however, and several alternative compounds with smart material capabilities play fundamental roles in the design of structures which can adapt or respond to external stimuli. Among currently employed materials, this includes polyvinylidene fluoride (PVDF) films, electrorheological and magnetorheological fluids, and various composites. The piezoelectric PVDF films are lightweight and compliant compared to PZT patches which limits their utility as structural actuators but provides them with unique sensor capabilities since they can be configured to target specific modes or structural responses. Details regarding the use of PVDF sensors in structural acoustic systems can be found in $[17,18]$. Electrorheological and magnetorheological fluids are characterized by reversible changes in the rheology of the fluids when subjected to electric or magnetic fields. This provides the capability for converting the fluids from low viscosity states to ones which are essentially solid. Potential applications which utilize these materials include vibration isolation systems such as active shock absorbers and engine mounts as well as electrically or magnetically triggered valves. Further applications employing these materials are described in $[9,33,47]$ while details concerning the modeling of electrorheological and magnetorheological fluids can be respectively found in $[48,66]$ and $[57,73,74]$.

As indicated throughout the review, the state of smart structures is rapidly evolving as new materials, models and control algorithms are developed. To optimize the capabilities of the materials in smart structure applications, it is necessary to consider material characterization, model development and control design in unison. This permits the development of models which quantify physical mechanisms pertinent to control implementation and the design of control techniques which capitalize on strengths of the smart material components and are commensurate with the models. The synergy between material characterization, model development and the design of smart structures which utilize these materials also extends to the design of new smart material compounds. For example, analysis of the fundamental mechanisms which produce the magnetomechanical coupling in Terfenol has led to the development of holmium alloys which exhibit reduced hysteresis [92].

To date, the majority of controllers for smart structures have been based on linear models and control laws. This can be attributed to the prevalence of PZT transducers, which exhibit quasilinear behavior at low to moderate drive levels, as well as the relative ease with which linear control laws 
can be implemented as compared with nonlinear methods. The introduction of new high performance materials and more stringent demands on existing materials, however, has led to the consideration of transducers which are inherently nonlinear. This necessitates the development of models and control laws which accommodate inherent hysteresis and constitutive nonlinearities. The consideration of nonlinear models and control methods is also required for applications which employ electrorheological and magnetorheological fluids due to the highly nonlinear dynamics associated with the materials. When characterizing the materials, it is advantageous to develop models which are applicable to a wide range of materials and can be inverted (at least approximately) to construct inverse compensators for linear control design. While nonlinear control laws can provide optimal performance, the ease of implementing linear controllers makes them preferable in the majority of current smart structure applications. Finally, the models and control methods will continue to evolve to accommodate the development of new smart materials and design of smart structures which utilize these materials.

\section{Acknowledgements}

The author thanks H.T. Banks, M.J. Dapino and D.J. Inman for reviewing the manuscript and providing input which helped to improve the presentation. This research was supported in part by the Air Force Office of Scientific Research through the grants AFOSR-F49620-98-1-0180 and AFOSRF49620-01-1-0107.

\section{References}

[1] A.A. Adly and I.D. Mayergoyz. "Magnetostriction simulation using anisotropic vector Preisachtype models." IEEE Transactions on Magnetics. 32(5), pp. 4773-4775, 1996.

[2] E.H. Anderson and N.W. Hagood. "Self-sensing piezoelectric actuation: Analysis and application to controlled structures." AIAA/ ASME/ASCE/AHS/ASC Structures, Structural Dynamics and Materials Conference, Dallas, TX, pp. 2141-2155, 1992.

[3] G. Avalos and I. Lasiecka. "Differential Riccati equation for the active control of a problem in structural acoustics." Journal of Optimization Theory and Applications. 91, pp. 695-728, 1996.

[4] H.T. Banks, W. Fang, R.J. Silcox and R.C. Smith. "Approximation methods for control of acoustic/structure models with piezoceramic actuators." Journal of Intelligent Material Systems and Structures. 4(1), pp. 98-116, 1993.

[5] H.T. Banks and K. Ito. "Approximation in LQR problems for infinite dimensional systems with unbounded input operators." Journal of Mathematical Systems, Estimation and Control. 7, pp. 134, 1997.

[6] H.T. Banks, A.J. Kurdila and G. Webb. "Identification of hysteretic control influence operators representing smart actuators Part I: Formulation." Mathematical Problems in Engineering. 3, pp. 287-328, 1997.

[7] H.T. Banks, A.J. Kurdila and G. Webb. "Identification of hysteretic control influence operators representing smart actuators Part II: Convergent approximations." Journal of Intelligent Material Systems and Structures. 8(6), pp. 536-550, 1997. 
[8] H.T. Banks, R.C. Smith, D.E. Brown, R.J. Silcox and V.L. Metcalf. "Experimental confirmation of a PDE-based approach to design of feedback controls." SIAM Journal on Control and Optimization. 35(4), pp. 1263-1296, 1997.

[9] H.T. Banks, R.C. Smith and Y. Wang. Smart Material Structures: Modeling, Estimation and Control. Masson/John Wiley, Paris/ Chichester, 1996.

[10] R.M. Bozorth. Ferromagnetism. IEEE Press, New York, 1978.

[11] W.F. Brown. Magnetoelastic Interactions. Springer-Verlag, Berlin, 1966.

[12] F.T. Calkins. "Design, analysis, and modeling of giant magnetostrictive transducers." PhD Dissertation, Iowa State University, Ames, IA, 1997.

[13] F.T. Calkins, R.C. Smith and A.B. Flatau. "An energy-based hysteresis model for magnetostrictive transducers," IEEE Transactions on Magnetics. 36(2), pp. 429-439, 2000.

[14] F.T. Calkins, R.L. Zrostlik and A.B. Flatau. "Terfenol-D vibration control of a rotating shaft." Proceedings of the 1994 ASME International Mechanical Engineering Congress and Exposition, Chicago IL; In Adaptive Structures and Composite Materials Analysis and Applications AD-Vol. 45, pp. 267-274, 1996.

[15] I-W. Chen and Y. Wang. "A domain wall model for relaxor ferroelectrics." Ferroelectrics. 206, pp. 245-263, 1998.

[16] A.E. Clark. "Magnetostrictive rare earth-Fe2 compounds." in Ferromagnetic Materials. Volume 1, E.P. Wohlfarth, Ed., North-Holland Publishing Company, Amsterdam, pp. 531-589, 1980.

[17] R.L. Clark, R.A. Burdisso and C.R. Fuller. "Design approaches for shaping polyvinylidene fluoride sensors in active structural acoustic control (ASAC)." Journal of Intelligent Material Systems and Structures. 4, pp. 354-365, 1993.

[18] R.L. Clark and C.R. Fuller. "Modal sensing of efficient acoustic radiators with polyvinylidene fluoride distributed sensors in active structural acoustic control approaches." Journal of the Acoustical Society of America. 91(6), pp. 3321-3329, 1992.

[19] R.L. Clark, W.R. Saunders and G.P. Gibbs. Adaptive Structures: Dynamics and Control. John Wiley and Sons, New York, 1998.

[20] D.G. Cole and R.L. Clark. "Adaptive compensation of piezoelectric sensoriactuators." Journal of Intelligent Material Systems and Structures. 5, pp. 665-672, 1994.

[21] L.E. Cross. "Recent developments in piezoelectric ferroelectric materials and composites." Proceedings of the 4th European and 2nd MIMR Conference, Ed., G.R. Tomlinson and W.A. Bullough, Harrogate, UK, July 6-8, pp. 89-97, 1998.

[22] B.D. Cullity. Introduction to Magnetic Materials. Addison-Wesley, Reading, MA, 1972.

[23] D. Damjanovic and R.E. Newnham. "Electrostrictive and piezoelectric materials for actuator applications." Journal of Intelligent Material Systems and Structures. 3, pp. 190-208, 1992.

[24] M.J. Dapino, F.T. Calkins and A.B. Flatau. "Magnetostrictive Devices." Wiley Encyclopedia of Electrical and Electronics Engineering. John G. Webster, Ed., John Wiley and Sons, Inc., Volume 12, pp. 278-305, 1999. 
[25] M.J. Dapino, R.C. Smith and A.B. Flatau. "An active and structural strain model for magnetostrictive transducers." Proceedings of the SPIE, Smart Structures and Integrated Systems, San Diego, CA, 1998, Vol. 3329, pp. 198-209.

[26] M.J. Dapino, R.C. Smith and A.B. Flatau. "A structural-magnetic strain model for magnetostrictive transducers," IEEE Transactions on Magnetics, 36(3), pp. 545-556, 2000.

[27] J. Dosch, D.J. Inman, and E. Garcia. "A self-sensing piezoelectric actuator for collocated control. Journal of Intelligent Material Systems and Structures. 3, pp. 166-185, 1992.

[28] T.A. Duenas, L. Hsu and G.P. Carman. "Magnetostrictive composite material systems analytical/experimental." Symp. Advances Smart Materials-Fundamentals Applications, Boston, MA, 1996.

[29] K. Elliot. "Titan vibroacoustics." Proceedings of the NASA-Industry Conference on Launch Environments of ELV Payloads, Elkridge, MD, June 1990, pp. 189-215.

[30] V.N. Fedosov and A.S. Sidorkin. "Quasielastic displacements of domain boundaries in ferroelectrics." Soviet Physics Solid State, 18(6), pp. 964-968, 1976.

[31] H. Funakubo. Shape Memory Alloys. Gordon and Breach, New York, 1986.

[32] W.S. Galinaitis and R.C. Rogers. "Control of a hysteretic actuator using inverse hysteresis compensation." SPIE Smart Structures and Materials, Mathematics and Control in Smart Structures, San Diego, CA, 1998.

[33] M.V. Gandhi and B.S. Thompson. Smart Materials and Structures. Chapman and Hall, London, 1992.

[34] P. Ge and M. Jouaneh. "Modeling hysteresis in piezoceramic actuators." Precision Engineering. 17, pp. 211-221, 1995.

[35] P. Ge and M. Jouaneh. "Tracking control of a piezoceramic actuator." IEEE Transactions on Control Systems Technology. 4(3), pp. 209-216, 1996.

[36] C.L. Hom and N. Shankar. "Modeling nonlinearity in electrostrictive sonar transducers." Journal of the Acoustical Society of America. 104(4), pp. 1903-1913, 1998.

[37] C.L. Hom and N. Shankar. "A constitutive model for relaxor ferroelectrics." SPIE Conference on Mathematics and Control in Smart Structures, Newport Beach, CA, Volume 3667, pp. 134-144, 1999 .

[38] F.V. Hunt. Electroacoustics: The Analysis of Transduction, and Its Historical Background. Acoustical Society of America, 1982.

[39] M.W. Hyer and A. Jilani. "Predicting the deformation characteristics of rectangular unsymmetrically laminated piezoelectric materials." Smart Materials and Structures, 7, pp. 1-8, 1998.

[40] P. Ioannou and J. Sun. Robust Adaptive Control. Prentice-Hall, Englewood Cliffs, NJ, 1996.

[41] B. Jaffe, W.R. Cook, Jr. and H. Jaffe. Piezoelectric Ceramics. Academic Press, New York, 1971.

[42] R.D. James and D. Kinderlehrer. "Theory of magnetostriction with applications to $\mathrm{Tb}_{x} \mathrm{Dy}_{1-x} \mathrm{Fe}_{2}$." Philosophical Magazine, B68, pp. 237-274, 1993. 
[43] R.D. James and M. Wuttig. "Ferromagnetic shape memory alloys." Proceedings of the SPIE, Smart Structures and Materials, San Diego, CA, Volume 3324, 1998.

[44] D.C. Jiles. Introduction to Magnetism and Magnetic Materials. Chapman and Hall, New York, 1991.

[45] D.C. Jiles and D.L. Atherton. "Theory of ferromagnetic hysteresis." Journal of Magnetism and Magnetic Materials. 61, pp. 48-60, 1986.

[46] D.C. Jiles and J.B. Thoelke. "Theoretical modelling of the effects of anisotropy and stress on the magnetization and magnetostriction of $\mathrm{Tb}_{0.3} \mathrm{Dy}_{0.7} \mathrm{Fe}_{2}$. Journal of Magnetism and Magnetic Materials. 134, pp. 143-160, 1994.

[47] M.R. Jolly, J.W. Bender and J.D. Carlson. "Properties and applications of commercial magnetorheological fluids." Proceedings of the SPIE, Smart Structures and Materials, San Diego, CA, pp. 262-275, 1998.

[48] G.M. Kamath and N.M. Wereley. "Nonlinear viscoelastic plastic model for electrorheological fluids." Smart Materials and Structures. 6(3), pp. 351-359, 1997.

[49] N.A. Koratkar and I. Chapra. "Testing and validation of a Froude scaled helicopter rotor model with piezo-bimorph actuated trailing edge flaps." Proceedings of the SPIE, Smart Structures and Integrated Systems, San Diego, CA, Vol. 3041, pp. 183-205, 1997.

[50] S.M. Kuo and D.R. Morgan. Active Noise Control Systems. Wiley, New York, 1996.

[51] A.J. Kurdila and G. Webb. "Compensation for distributed hysteresis operators in active structural systems." Journal of Guidance, Control, and Dynamics. 20(6), pp. 1133-1140, 1997.

[52] H. Kwakernaak and R. Sivan. Linear Optimal Control System. John Wiley \& Sons, Inc., New York, 1972.

[53] D. Lagoudas, Z. Bo and M. Qidwai. "A unified thermodynamic constitutive model for SMA and finite element analysis of active metal matrix composites." Mechanics of Composite Structures. 3(2), pp. 153-179, 1996.

[54] B.D. Laikhtman. "Flexural vibrations of domain walls and dielectric dispersion of ferroelectrics." Soviet Physics Solid State. 15(1), pp. 62-68, 1973.

[55] I. Lasiecka. "Finite element approximations of compensator design for analytic generators with fully unbounded controls/observations." SIAM Journal of Control and Optimization. 33(1), pp. 6788, 1995.

[56] C. Liang and C.A. Rogers. "A one-dimensional thermomechanical constitutive relation of shape memory materials." Journal of Intelligent Material Systems and Structures. 1(2), pp. 207-234, 1990.

[57] H.V. Ly, R. Reitich, M.R. Jolly, H.T. Banks and K. Ito. "Simulations of particle dynamics in magnetorheological fluids." Journal of Computational Physics. 155, pp. 160-177, 1999.

[58] J.A. Main, E. Garcia and D.V. Newton. "Precision position control of piezoelectric actuators using charge feedback." Journal of Guidance, Control and Dynamics. 18(5), pp. 1068-1073, 1995. 
[59] J.E. Miesner and J.P. Teter. "Piezoelectric/magnetostrictive resonant inchworm motor." Proceedings of SPIE, Smart Structures and Materials, Volume 2190, pp. 520-527, 1994.

[60] K.M. Mossi, G.V. Selby and R.G. Bryant. "Thin-layer composite unimorph ferroelectric driver and sensor properties." Materials Letters. 35, pp. 39-49, 1998.

[61] A.J. Moulson and J.M. Herbert. Electroceramics: Materials, Properties, Applications. Chapman and Hall, New York, 1990.

[62] P.A. Nelson and S.J. Elliott. Active Control of Sound. Academic Press, New York, 1992.

[63] R.E. Newnham. "Electroceramics." Reports on Progress in Physics. 52, pp. 123-156, 1989.

[64] J.F. Nye. Physical Properties of Crystals: Their Representation by Tensors and Matrices. Oxford Press, London. 1957.

[65] M. Omura, H. Adachi and Y. Ishibashi. "Simulations of ferroelectric characteristics using a onedimensional lattice model." Japanese Journal of Applied Physics. 30(9B), pp. 2384-2387, 1991.

[66] M. Parthasarathy and D.J. Klingenberg. "Electrorheology: mechanisms and models." Material Science and Engineering. R17, pp. 57-103, 1996.

[67] J. Piquette and S.E. Forsythe. "A nonlinear material model of lead magnesium niobate (PMN)." Journal of the Acoustical Society of America. 101(1), pp. 289-296, 1997.

[68] P.M. Prenter. Splines and Variational Methods. Wiley, New York, 1975.

[69] J.B. Restorff. "Magnetostrictive materials and devices." Encyclopedia of Applied Physics, Vol. 9, pp. 229-244, 1994.

[70] J.B. Restorff, H.T. Savage, A.E. Clark and M. Wun-Fogle. "Preisach modeling of hysteresis in Terfenol-D." Journal of Applied Physics. 67(9), pp. 5016-5018, 1996.

[71] N.N. Rogacheva. The Theory of Piezoelectric Shells and Plates. CRC Press, Boca Raton, FL, 1994.

[72] R.L. Sarno and M.E. Franke. "Suppression of flow induced pressure oscillations in cavities." Journal of Aircraft. 31(1), pp. 90-96, 1994.

[73] T.M. Simon, F. Reitich, M.R. Jolly, K. Ito and H.T. Banks. "Estimation of the effective permeability in magnetorheological fluids." CRSC Technical Report CRSC-TR98-35; Journal of Intelligent Material Systems and Structures, submitted.

[74] T.M. Simon, F. Reitich, M.R. Jolly, K. Ito and H.T. Banks. "On the effective magnetic properties of magnetorheological fluids." CRSC Technical Report CRSC-TR98-42; Mathematical and Computer Modeling, to appear.

[75] R.C. Smith, "Modeling techniques for magnetostrictive actuators." Proceedings of the SPIE, Smart Structures and Materials 1997: Smart Structures and Integrated Systems, San Diego, CA, Vol. 3041, pp. 243-253, 1997.

[76] R.C. Smith. "Hysteresis modeling in magnetostrictive materials via Preisach operators." Journal of Mathematical Systems, Estimation and Control, 8(2), summary pp. 249-252, 1998. 
[77] R.C. Smith. "A nonlinear optimal control method for magnetostrictive actuators." Journal of Intelligent Material Systems and Structures. 9(6), pp. 468-486, 1998.

[78] R.C. Smith. "Inverse compensation for hysteresis in magnetostrictive transducers." Mathematical and Computer Modelling, 33, pp. 285-298, 2001.

[79] R.C. Smith and C.L. Hom. "A domain wall model for ferroelectric hysteresis." SPIE Conference on Mathematics and Control in Smart Structures, Volume 3667, Newport Beach, CA, pp. 150-161, 1999 .

[80] R.C. Smith and C.L. Hom. "A domain wall theory for ferroelectric hysteresis." Journal of Intelligent Material Systems and Structures, 10(3), pp. 195-213, 1999.

[81] R.C. Smith and Z. Ounaies. "A hysteresis model for piezoceramic materials." CRSC Technical Report CRSC-TR99-21; Proceedings of the ASME International Mechanical Engineering Congress and Exposition, Nashville, TN, November, 1999.

[82] R.C. Smith and Z. Ounaies. "A domain wall model for hysteresis in piezoelectric materials." Journal of Intelligent Material Systems and Structures, 11(1), pp. 62-79, 2000.

[83] R.C. Smith and R. Zrostlik. "Inverse compensation for ferromagnetic hysteresis." Proceedings of the 1999 IEEE Conference on Decision and Control, Phoenix, AZ, December 7-10, 1999.

[84] E.C. Stoner and E.P. Wohlfarth. "A mechanism of magnetic hysteresis in heterogeneous alloys." Philosophical Transactions of the Royal Society of London. A240, pp. 599-642, 1948.

[85] G. Tao and P.V. Kokotović. Adaptive Control of Systems with Actuator and Sensor Nonlinearities. John Wiley and Sons, New York, 1996.

[86] R. Venkataraman. "A hybrid actuator." M.S. Thesis, University of Maryland, 1995.

[87] G. Webb, L. Wilson, D. Lagoudas and O. Rediniotis. "Control of SMA actuators in dynamic environments." Proceedings of the SPIE Conference on Mathematics and Control in Smart Structures, Newport Beach, CA, pp. 278-289, 1999.

[88] B. Widrow and M.E. Hoff, Jr. "Adaptive switching circuits." IRE WESCON, Conv. Rec. Pt 4, pp. 96-104, 1960.

[89] B. Widrow and S.D. Stearns. Adaptive Signal Processing. Prentice Hall, Englewood Cliffs, NJ, 1985.

[90] B. Widrow and E. Walach. Adaptive Inverse Control. Prentice Hall, NJ, 1996.

[91] J.M. Wiltse and A. Glezer. "Manipulation of free shear flows using piezoelectric actuators." Journal of Fluid Mechanics. 249, pp. 261-285, 1993.

[92] M. Wun-Fogle, J.B. Restorff, A.E. Clark and J.F. Lindberg. "Magnetization and magnetostriction of dendritic [112] $\mathrm{Tb}_{x} \mathrm{Dy}_{y} \mathrm{Ho}_{z} \mathrm{Fe}_{1.95}(x+y+z=1)$ rods under compressive stress." Journal of Applied Physics. 83(11), pp. 7279-7281, 1998. 